\title{
ELLIPTIC AND PARABOLIC REGULARITY FOR SECOND ORDER DIVERGENCE OPERATORS WITH MIXED BOUNDARY CONDITIONS
}

\author{
ROBERT HALLER-DINTELMANN, ALF JONSSON, DOROTHEE KNEES, AND JOACHIM REHBERG
}

\begin{abstract}
We study second order equations and systems on non-Lipschitz domains including mixed boundary conditions. The key result is interpolation for suitable function spaces. From this, elliptic and parabolic regularity results are deduced by means of Sneiberg's isomorphism theorem.
\end{abstract}

\section{INTRODUCTION}

In this paper we first establish interpolation properties for function spaces that are related to mixed boundary value problems. Afterwards, from this and a fundamental result of Sneiberg [59] (cf. also [63]) we deduce elliptic and parabolic regularity results for second order, divergence operators.

In recent years it became manifest that the appearance of mixed boundary conditions is not the exception when modelling real world problems, but more the rule. For instance, in semiconductor theory, models with only pure Dirichlet or pure Neumann conditions are meaningless, see [57].

One geometric concept, which proved of value for the analysis of mixed boundary value problems, is that introduced by Gröger in [26] (compare also [55] and references therein). It demands, roughly speaking, that the domain $\Omega$ under consideration is a Lipschitz domain and that the 'Dirichlet part' $D \subset \partial \Omega$ of the boundary is locally separated from the rest by a Lipschitzian hypersurface within $\partial \Omega$. Within this geometric framework, several properties for differential operators, well-known from smooth constellations, were re-established. This concerns elliptic regularity (in particular Hölder continuity) [26, [22, 25], 28, maximal parabolic regularity 23, [29, 31] and interpolation [24].

In this paper, we impose more general conditions on the domain and on the Dirichlet boundary part $D$; notably, we dispense the Lipschitz property of the domain. In particular, the domain may touch itself from outside, see the examples in Figures 1 and 2 below that are included in our framework. Note that the situation in Figure 2 is not an artificial one: the reader may think of a body for which $\Sigma$ and the two striped areas form an extremely thin, but highly conducting contact $D$, to which an external source (e.g. heat or electrical) is applied. If the body is formed by a much less conducting material, the distribution of heat/charge within the body is subject of an elliptic/parabolic equation with Dirichlet conditions on $D$.

Our geometric framework is the following. The Dirichlet boundary part $D$ only has to be a $(d-1)$-set in the sense of Jonsson/Wallin. This can be seen as an - extremely weak - compatibility condition between $D$ and $\partial \Omega \backslash D$. For the complement of the Dirichlet boundary part, the crucial feature is the local extendability of Sobolev functions. Within this geometrical framework we prove the following: the spaces $W_{D}^{1, p}(\Omega)$ (cf. Definition $\left.2.3 p, p \in\right] 1, \infty$ [ interpolate according to the same rules as if one formally replaces the domain $\Omega$ by a ball $B$ and the boundary part $D \subset \partial \Omega$ by the empty set (compare [61, Ch. 4.3.1]). Based on the interpolation results we reproduce Gröger's elliptic regularity result from [26, namely that an arbitrary elliptic divergence operator

2010 Mathematics Subject Classification. 35B65, 35J47, 35J57, 74B05.

Key words and phrases. mixed boundary conditions, interpolation, elliptic regularity for equations and systems, analytic semigroups . 


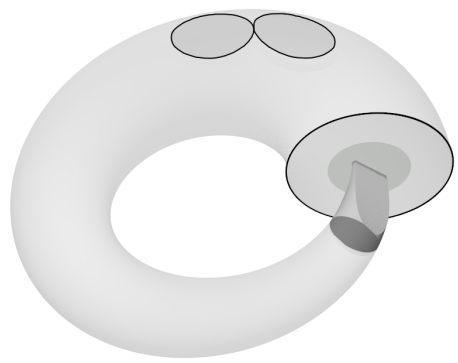

Figure 1. A geometric non-Lipschitzian setting which fulfills our assumptions, if the grey apex and the three shaded circles carry the Dirichlet condition.

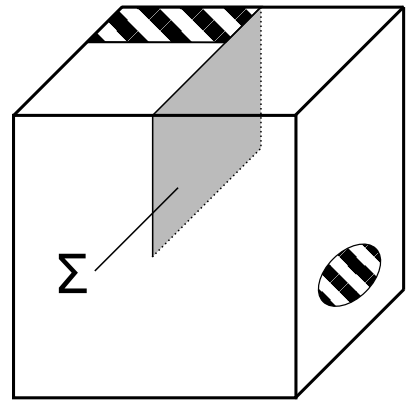

Figure 2. The set $\Sigma$ does not belong to $\Omega$, and carries - together with the striped parts - the Dirichlet condition.

$-\nabla \cdot \mu \nabla+1$ provides a topological isomorphism between a space $W_{D}^{1, p}(\Omega)$ and $W_{D}^{-1, p}(\Omega)$ for $p$ close to 2 - but now for a much broader class of domains and Dirichlet boundary parts. Let us emphasize that the - matrix valued - coefficient function $\mu$ of the operator needs only be bounded and elliptic, cf. Assumption 5.4 below. Note that the main result from [26] was used in some tens of papers in order to treat (mostly two dimensional) problems, stemming from real world applications. Having this regularity result at hand, we succeed in proving that divergence operators of this type generate analytic semigroups on spaces $W_{D}^{-1, p}(\Omega)$, as long as $p$ is chosen close to 2. Clearly, this can serve as the adequate instrument for the treatment of corresponding parabolic problems, compare e.g. [1, 32, Ch. 2], 47].

One of our main technical tools is the version of the now classical restriction/extension theorem of Jonsson/Wallin ([38, Ch. V.1 Thm.1]) for the limit case of Lipschitz functions, see Proposition 2.4 below.

Throughout we stick to the condition that $D$ is a $d-1$-set, which in several instances can in fact be weakened. Since our motivation for this paper comes from the applications, our aim is to describe a very general but nevertheless easily accessible geometric constellation that allows to deduce our results.

The outline of the paper is as follows: in the next section we introduce some preliminaries. In Section 3 we reproduce interpolation within the family of spaces $\left\{W_{D}^{1, p}(\Omega)\right\}_{p \in] 1, \infty[}$, and, as a

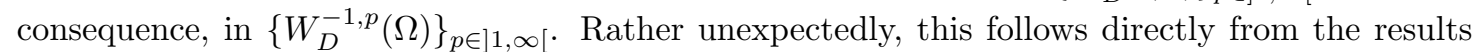
of Jonsson/Wallin, combined with a classical interpolation principle for complemented subspaces and the existence of an extension operator $\mathfrak{E}: W_{D}^{1, p}(\Omega) \rightarrow W_{D}^{1, p}\left(\mathbb{R}^{d}\right)$, which is uniform in $p$.

Since the existence of an extension operator is thus crucial for our approach, in Section 4 we first establish construction principles for extension operators. These, together with our conditions on the geometry of $\Omega$ near $\partial \Omega \backslash D$, then indeed assure their existence. A simple 'pre-processing', which essentially improves the applicability of our setting, is described in Lemmas 4.1 and 4.3 . It allows to pass from the original domain $\Omega$ to another domain $\Omega_{\bullet} \subseteq \bar{\Omega}$ whose boundary is smaller and, in most cases, a more regular one. It is exactly this what enables also the treatment of geometric settings like in Figure 2, compare 44 for a similar, but different approach - there even applied to higher order Sobolev spaces. Section 5 contains the above mentioned elliptic and parabolic regularity results. In Section 6 we extend the discussion to a class of elliptic systems comprising the equations for linear elasticity and for Cosserat models. Relying on the interpolation results it 
is shown that the corresponding differential operators provide topological isomorphisms between $\mathbb{W}_{D}^{1, p}(\Omega)$ and $\mathbb{W}_{D}^{-1, p}(\Omega)$ for suitable $p>2$. Moreover, under an additional symmetry assumption on the coefficient tensor, uniform estimates are derived for classes of coefficient tensors satisfying certain uniform bounds. Since in the case of systems the coercivity of the operator not necessarily entails the positivity of the coefficient tensor, the pointwise arguments from 26 have to be modified and transferred to arguments dealing with the whole operator. In this way also the results from 33 are extended to more general geometric situations.

Finally, in Section 7. we point out a broad class of possible applications for our regularity results.

\section{Notation, Preliminaries}

If $X$ and $Y$ are two Banach spaces, then we use the symbol $\mathcal{L}(X ; Y)$ for the space of linear, continuous operators from $X$ to $Y$. In case of $X=Y$ we abbreviate $\mathcal{L}(X)$.

We are now going to impose the adequate condition on the Dirichlet boundary part $D$. For this we first recall the notion of an $l$-set, cf. Jonsson/Wallin [38, II.1.1/2].

Definition 2.1. Assume $0<l \leq d$. Let $M \subset \mathbb{R}^{d}$ be closed and $\rho$ the restriction of the $l$ dimensional Hausdorff measure $\mathcal{H}_{l}$ to $M$. Then $M$ is called an $l$-set, if there exist two positive constants $c_{1}, c_{2}$ that satisfy

$$
\left.c_{1} r^{l} \leq \rho(B(\mathrm{x}, r) \cap M) \leq c_{2} r^{l}, \quad \mathrm{x} \in M, r \in\right] 0,1[,
$$

where $B(\mathrm{x}, r)$ is the ball with center $\mathrm{x}$ and radius $r$ in $\mathbb{R}^{d}$.

Assumption 2.2. Let $\Omega \subset \mathbb{R}^{d}$ always be a bounded domain and let $\Gamma$ be an open part of $\partial \Omega$, such that $D:=\partial \Omega \backslash \Gamma$ is a $(d-1)$-set.

We now define the adequate Sobolev space of first order that reflects the Dirichlet condition.

Definition 2.3. Let $\Lambda \subset \mathbb{R}^{d}$ be a domain and let $F$ be a closed subset of $\bar{\Lambda}$. Then we define

$$
C_{F}^{\infty}(\Lambda):=\left\{\left.\psi\right|_{\Lambda}: \psi \in C^{\infty}\left(\mathbb{R}^{d}\right), \operatorname{supp}(\psi) \cap F=\emptyset\right\} .
$$

Moreover, for $p \in\left[1, \infty\left[\right.\right.$, we denote the closure of $C_{F}^{\infty}(\Lambda)$ in $W^{1, p}(\Lambda)$ by $W_{F}^{1, p}(\Lambda)$.

In particular, the set $F$ may be identical with the boundary part $D$.

Since the ultimate instrument for almost everything in the next section is a classical result of Jonsson/Wallin (see [38, Ch. VII]) we quote this here for the convenience of the reader.

Proposition 2.4. Let $F \subset \mathbb{R}^{d}$ be closed and, additionally, a $(d-1)$-set.

(i) There is a continuous restriction operator $\mathcal{R}_{F}$ which maps every space $W^{1, p}\left(\mathbb{R}^{d}\right)$ continuously onto the Besov space $B_{p, p}^{1-\frac{1}{p}}(F)$ as long as $\left.p \in\right] 1, \infty[$.

(ii) Conversely, there is an extension operator $\mathcal{E}_{F}$ which maps each space $B_{p, p}^{1-\frac{1}{p}}(F)$ continuously into $W^{1, p}\left(\mathbb{R}^{d}\right)$, provided $\left.p \in\right] 1, \infty[$.

(iii) By construction, $\mathcal{E}_{F}$ is a right inverse for $\mathcal{R}_{F}$, i.e. $\mathcal{R}_{F} \mathcal{E}_{F}$ is the identity operator on $B_{p, p}^{1-\frac{1}{p}}(F), c f$. [38, Ch.V.1.3].

It turns out that the extension operator $\mathcal{E}_{F}$ even maintains Lipschitz continuity:

Theorem 2.5. Let $F \subset \mathbb{R}^{d}$ be closed and, additionally, a $(d-1)$-set. Then the operator $\mathcal{E}_{F}$ from Proposition 2.4 maps the space of Lipschitz continuous functions on F continuously into the space of Lipschitz continuous functions on $\mathbb{R}^{d}$.

Proof. The extension operator $\mathcal{E}_{F}$ is of Whitney type, and we need some facts about the Whitney decomposition of $\mathbb{R}^{d} \backslash F$ and a related partition of unity $\left\{\phi_{i}\right\}$, cf. 38. for more background and 
details. The decomposition is a collection of closed, dyadic cubes $Q_{i}$, with sidelength $2^{N_{i}}$ for integers $N_{i}$, and with mutually disjoint interiors, such that $\bigcup Q_{i}=\mathbb{R}^{d} \backslash F$, and

$$
\operatorname{diam} Q_{i} \leq \mathrm{d}\left(Q_{i}, F\right) \leq 4 \operatorname{diam} Q_{\mathrm{i}},
$$

where $\mathrm{d}\left(Q_{i}, F\right)$ is the distance between $Q_{i}$ and $F$. Denote the diameter of $Q_{i}$ by $l_{i}$, its sidelength by $s_{i}$, and its center by $\mathrm{x}_{i}$, and let $Q_{i}^{\star}$ denote the cube obtained by expanding $Q_{i}$ around its center with a factor $\iota, 1<\iota<5 / 4$. It follows from $(2.3)$ that

$$
1 / 4 l_{i} \leq l_{k} \leq 4 l_{i}
$$

if $Q_{i}$ and $Q_{k}$ touch. This means that $Q_{i}^{\star}$ intersects a cube $Q_{k}$ only if $Q_{i}$ and $Q_{k}$ touch, and that each point in $\mathbb{R}^{d} \backslash F$ is contained in at most $N_{0}$ cubes $Q_{i}^{\star}$, where $N_{0}$ is a number depending only on the dimension $d$.

Next, nonnegative $C^{\infty}$-functions $\phi_{i}$ are chosen in such a way that $\phi_{i}(\mathrm{x})=0$ if $\mathrm{x} \notin Q_{i}^{\star}$, $\sum_{i} \phi_{i}(\mathrm{x})=1, \mathrm{x} \in \mathbb{R}^{d} \backslash F$, and so that $\left|D^{j} \phi_{i}\right| \leq c l_{i}^{-|j|}$ for any $j$, where $c$ depends on $j$.

Let $I$ denote those $i$ such that $s_{i} \leq 1$, let $\rho$ again be the restriction of the $(d-1)$-dimensional Hausdorff measure on $F$, and put $c_{i}=\rho\left(B\left(\mathrm{x}_{i}, 6 l_{i}\right)\right)^{-1}$. Note that it follows from (2.1) and (2.3), that $\rho\left(B\left(\mathrm{x}_{i}, 6 l_{i}\right)\right)>0$. The extension operator used in Proposition 2.4 is given by

$$
\mathcal{E}_{F} f(\mathrm{x})=\sum_{i \in I} \phi_{i}(\mathrm{x}) c_{i} \int_{\left|\mathrm{t}-\mathrm{x}_{i}\right| \leq 6 l_{i}} f(\mathrm{t}) \mathrm{d} \rho(\mathrm{t}), \quad \mathrm{x} \in \mathbb{R}^{d} \backslash F,
$$

and $\mathcal{E}_{F} f(\mathrm{x})=f(\mathrm{x})$ for $\mathrm{x} \in F$.

We now head for Lipschitz continuity of $\mathcal{E}_{F} f$. To begin with let $\mathrm{x}$ and y be in cubes with sides $\leq 1 / 4$. Then $\sum \phi_{i}(\mathrm{x})=\sum \phi_{k}(\mathrm{y})=1$, where the sums are taken over all $i$ and $k$, respectively. Using this, one obtains, for any constant $b$,

$$
\mathcal{E}_{F} f(\mathrm{x})-b=\sum_{i} \phi_{i}(\mathrm{x}) c_{i} \int_{\left|\mathrm{t}-\mathrm{x}_{i}\right| \leq 6 l_{i}}(f(\mathrm{t})-b) \mathrm{d} \rho(\mathrm{t}),
$$

and taking $b=\mathcal{E}_{F} f(\mathrm{y})$

$$
\mathcal{E}_{F} f(\mathrm{x})-\mathcal{E}_{F} f(\mathrm{y})=\sum_{i} \sum_{k} \phi_{i}(\mathrm{x}) \phi_{k}(\mathrm{y}) c_{i} c_{k} \iint_{\left|\mathrm{t}-\mathrm{x}_{i}\right| \leq 6 l_{i},\left|\mathrm{~s}-\mathrm{x}_{k}\right| \leq 6 l_{k}}(f(\mathrm{t})-f(\mathrm{~s})) \mathrm{d} \rho(\mathrm{t}) \mathrm{d} \rho(\mathrm{s}) .
$$

We also have

$$
D^{j}\left(\mathcal{E}_{F} f\right)(\mathrm{x})=\sum_{i} D^{j} \phi_{i}(\mathrm{x}) c_{i} \int_{\left|\mathrm{t}-\mathrm{x}_{i}\right| \leq 6 l_{i}} f(\mathrm{t}) \mathrm{d} \rho(\mathrm{t})
$$

and, for $|j|>0$, since then $\sum_{i} D^{j} \phi_{i}(\mathrm{x})=0$, so we can subtract $\mathcal{E}_{F} f(\mathrm{y})$ from the integrand,

$$
D^{j}\left(\mathcal{E}_{F} f\right)(\mathrm{x})=\sum_{i} \sum_{k} D^{j} \phi_{i}(\mathrm{x}) \phi_{k}(\mathrm{y}) c_{i} c_{k} \iint_{\left|\mathrm{t}-\mathrm{x}_{i}\right| \leq 6 l_{i},\left|\mathrm{~s}-\mathrm{x}_{k}\right| \leq 6 l_{k}}(f(\mathrm{t})-f(\mathrm{~s})) \mathrm{d} \rho(\mathrm{t}) \mathrm{d} \rho(\mathrm{s}) .
$$

Assume now that $f$ is Lipschitz continuous with Lipschitz norm 1. Let $\mathrm{x} \in Q_{\nu}, \mathrm{y} \in Q_{\eta}$, where, say, $s_{\nu} \geq s_{\eta}$, and assume first $s_{\nu} \leq 1 / 4$. If $|\mathrm{x}-\mathrm{y}|<l_{\nu} / 2$, then by the mean value theorem $\mathcal{E}_{F} f(\mathrm{x})-\mathcal{E}_{F} f(\mathrm{y})=\nabla\left(\mathcal{E}_{F} f\right)(\xi) \cdot(\mathrm{x}-\mathrm{y})$ for some $\xi$ with $|\mathrm{x}-\xi|<l_{\nu} / 2$. Note that the geometric constellation assures that the whole segment joining $\mathrm{x}$ and $\mathrm{y}$ avoids $F$, so the mean value theorem is applicable. Next, take $\kappa$ so that $\xi \in Q_{\kappa}$. Now we use, if $s_{\kappa} \leq 1 / 4$ (otherwise, see below), 2.9 with $\mathrm{x}$ and $\mathrm{y}$ equal to $\xi$, and recall that if $\phi_{i}(\xi) \neq 0$, then $Q_{i}$ and $Q_{\kappa}$ touch. For nonzero terms we then have, for $\mathrm{t}$ and $\mathrm{s}$ in the domain of integration, $|\mathrm{t}-\mathrm{s}| \leq\left|\mathrm{t}-\mathrm{x}_{i}\right|+\left|\mathrm{x}_{i}-\mathrm{x}_{\kappa}\right|+\left|\mathrm{x}_{\kappa}-\mathrm{x}_{k}\right|+\left|\mathrm{x}_{k}-s\right| \leq 7 l_{i}+2 l_{\kappa}+7 l_{k}$, and also, by (2.4), that, $l_{i}$ and $l_{k}$ are comparable to $l_{\kappa}$. Recalling that $0 \leq \phi_{i} \leq 1,\left|D^{j} \phi_{i}\right| \leq c l_{i}^{-1}$ for $|j|=1$ and using $|f(\mathrm{~s})-f(\mathrm{t})| \leq|\mathrm{t}-\mathrm{s}|$, one immediately obtains $\left|D^{j}\left(\mathcal{E}_{F} f\right)(\xi)\right| \leq c$ for $|j|=1$, so

$$
\left|\mathcal{E}_{F} f(\mathrm{x})-\mathcal{E}_{F} f(\mathrm{y})\right| \leq c|\mathrm{x}-\mathrm{y}| .
$$


If $|\mathrm{x}-\mathrm{y}| \geq l_{\nu} / 2$, we use (2.7) together with the observation that now $|\mathrm{t}-\mathrm{s}| \leq\left|\mathrm{t}-\mathrm{x}_{i}\right|+\left|\mathrm{x}_{i}-\mathrm{x}\right|+$ $|\mathrm{x}-\mathrm{y}|+\left|\mathrm{y}-\mathrm{y}_{k}\right|+\left|\mathrm{y}_{k}-\mathrm{s}\right| \leq 7 l_{i}+l_{\nu}+l_{\eta}+7 l_{k}+|\mathrm{x}-\mathrm{y}| \leq 58 l_{\nu}+|\mathrm{x}-\mathrm{y}| \leq c|\mathrm{x}-\mathrm{y}|$ if $\phi(\mathrm{x})$ and $\phi(\mathrm{y})$ are nonzero, and obtain again (2.10). If instead $\mathrm{y} \in F$ we get the same result using (2.6) with $b=f(\mathrm{y})$ and $|\mathrm{t}-\mathrm{y}| \leq\left|\mathrm{t}-\mathrm{x}_{i}\right|+\left|\mathrm{x}_{i}-\mathrm{x}\right|+|\mathrm{x}-\mathrm{y}| \leq 7 l_{i}+l_{\nu}+|\mathrm{x}-\mathrm{y}| \leq c|\mathrm{x}-\mathrm{y}|$, since, by (2.3), $l_{\nu} \leq|\mathrm{x}-\mathrm{y}|$.

If $s_{\nu}>1 / 4$, or $s_{\kappa}>1 / 4$, we can no longer use (2.9), 2.7), and (2.6). In the case $|\mathrm{x}-\mathrm{y}|<l_{\nu} / 2$, (2.8) together with $|f| \leq 1$ gives the desired estimate $\left|D^{j}\left(\mathcal{E}_{F} f\right)(\xi)\right| \leq c l_{\kappa}^{-1} \leq c$ for $|j|=1$. Using (2.5) we see that $\left|\mathcal{E}_{F} f\right| \leq c$ everywhere, which in particular implies $(2.10)$ in the remaining cases.

Remark 2.6. $\quad$ (i) Since the detailed structure of the Besov spaces $B_{p, p}^{1-\frac{1}{p}}(F)$ is not of interest in this paper, we refer to [38, Ch. V.1] for a definition.

(ii) It is known that, for any $f \in W^{1, p}\left(\mathbb{R}^{d}\right)$,

$$
\lim _{r \rightarrow 0} \frac{1}{|B(\mathrm{y}, r)|} \int_{B(\mathrm{y}, r)} f(\mathrm{x}) d \mathrm{x}
$$

exists for $\mathcal{H}_{d-1}$-almost all $\mathrm{y} \in \mathbb{R}^{d}$ (even more is true, see 65, Ch. 3.1]). Moreover, the function, defined by (2.11), reproduces $f$ within its Sobolev class, and the restriction of $f$ to any $(d-1)$-set $F$ is established this way, compare [38, Ch. 2.1].

(iii) The proof of Theorem 2.5 does in fact not require much about the measure $\rho$. The only thing needed is that the measure of any ball with center in $F$ is positive, which in particular holds for any $l$-measure with $0<l \leq n$.

For all what follows we fix an open ball $B$ which contains $\bar{\Omega}$. In the sequel we consider in our case $F=D$ the restriction/extension operators $\mathcal{R}_{F} / \mathcal{E}_{F}$ not only on all of $\mathbb{R}^{d}$, but also on the ball $B$. Since $D \subset B$ and the restriction operator $\mathcal{R}_{D}$ takes into account only the local behaviour of functions near $D$, the operator $\mathcal{E}_{D}$ remains a right inverse of $\mathcal{R}_{D}$ in this understanding. In this spirit, we also maintain the notations $\mathcal{E}_{D}, \mathcal{R}_{D}$.

Definition 2.7. If $\Lambda \subseteq \mathbb{R}^{d}$ is a domain and $F \subset \Lambda$ is a $(d-1)$-set, then we write

$$
\mathcal{W}_{F}^{1, p}(\Lambda):=\left\{\psi \in W^{1, p}(\Lambda): \mathcal{R}_{F} \psi=0 \text { a.e. on } F\right\},
$$

where the measure on $F$ is again $\left.\mathcal{H}_{d-1}\right|_{F}$, cf. Definition 2.1

It is a natural question whether $\mathcal{W}_{F}^{1, p}(\Lambda)=W_{F}^{1, p}(\Lambda)$ holds. An affirmative answer will be given in Corollary 3.7 - which will serve as a technical tool for the proof of the interpolation results below.

\section{INTERPOLATION}

In this section we establish interpolation results that are well-known for $\mathbb{R}^{d}$ or smooth domains, for the spaces $W_{D}^{1, p}(\Omega)$. As already mentioned in the introduction, the crucial ingredient is a Sobolev extension operator. So we introduce the following assumption.

Assumption 3.1. There exists a linear, continuous extension operator $\mathfrak{E}: W_{D}^{1,1}(\Omega) \rightarrow W_{D}^{1,1}\left(\mathbb{R}^{d}\right)$ which simultaneously defines a continuous extension operator $\mathfrak{E}: W_{D}^{1, p}(\Omega) \rightarrow W_{D}^{1, p}\left(\mathbb{R}^{d}\right)$ for every $p \in] 1, \infty[$.

Remark 3.2. (i) We are aware that Assumption 3.1 is of quite different character in comparison to Assumption 2.2. Only by formulating the results in this abstract way, it becomes manifest that it is only the functorial property of the extension operator that carries over the interpolation results. However, in Section 4 we will subsequently establish geometric conditions on $\Omega$ and $D$ that will assure Assumption 3.1 
(ii) Combining the mapping $\mathfrak{E}$ with the operator that restricts any function on $\mathbb{R}^{d}$ to $B$, one obtains an operator that maps $W_{D}^{1, p}(\Omega)$ continuously into the space $W_{D}^{1, p}(B)$; we maintain the notation $\mathfrak{E}$ for the resulting operator.

(iii) Under Assumptoin 3.1, one can establish the corresponding Sobolev embeddings $W_{D}^{1, p}(\Omega) \rightarrow$ $L^{q}(\Omega)$ (compactness, included) in a straightforward manner.

Our main result on interpolation is the following.

Theorem 3.3. Let Assumptions 2.2 and 3.1 be satisfied. Then complex and real interpolation

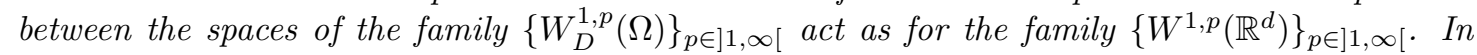
particular, one has for $\left.p_{0}, p_{1} \in\right] 1, \infty\left[\right.$ and $\frac{1}{p}=\frac{1-\theta}{p_{0}}+\frac{\theta}{p_{1}}$

$$
\left[W_{D}^{1, p_{0}}(\Omega), W_{D}^{1, p_{1}}(\Omega)\right]_{\theta}=W_{D}^{1, p}(\Omega)=\left(W_{D}^{1, p_{0}}(\Omega), W_{D}^{1, p_{1}}(\Omega)\right)_{\theta, p},
$$

Corollary 3.4. Let $\hat{W}_{D}^{-1, q}(\Omega)$ denote the dual of $W_{D}^{1, q^{\prime}}(\Omega), \frac{1}{q}+\frac{1}{q^{\prime}}=1$ and $W_{D}^{-1, q}(\Omega)$ denote the space of continuous antilinear forms on $W_{D}^{1, q^{\prime}}(\Omega), \frac{1}{q}+\frac{1}{q^{\prime}}=1$. For $\left.p_{0}, p_{1} \in\right] 1, \infty\left[\right.$ and $\frac{1}{p}=\frac{1-\theta}{p_{0}}+\frac{\theta}{p_{1}}$ one has

$$
\left[\hat{W}_{D}^{-1, p_{0}}(\Omega), \hat{W}_{D}^{-1, p_{1}}(\Omega)\right]_{\theta}=\hat{W}_{D}^{-1, p}(\Omega)
$$

and

$$
\left[W_{D}^{-1, p_{0}}(\Omega), W_{D}^{-1, p_{1}}(\Omega)\right]_{\theta}=W_{D}^{-1, p}(\Omega)
$$

Proof. Concerning (3.1), one employs the duality formula for complex interpolation in case of reflexive Banach spaces (see [61, Ch. 1.11.3]), which reads as $\left[X^{\prime}, Y^{\prime}\right]_{\theta}=[X, Y]_{\theta}^{\prime}$. In order to get (3.2), one associates to any linear form $T$ an antilinear form $T_{a}$ defining $\left\langle T_{a}, \psi\right\rangle:=\langle T, \bar{\psi}\rangle$. It is clear that the mappings $T \mapsto T_{a}$ and $T_{a} \mapsto T$ form a retraction/coretraction pair, thus (3.2) may be derived from 3.1 by the retraction/coretraction theorem for interpolation.

Theorem 3.3 will be proved in two steps. First we establish the corresponding result for the spaces $W_{F}^{1, p}(B)$ where $B$ is a ball and $F \subseteq B$ is a $(d-1)$-set. From this we will then deduce the general statement.

One main ingredient is the Jonsson/Wallin result from Proposition 2.4 and Theorem 2.5. We use this in the following way: the right inverse property of $\mathcal{E}_{F}$ for $\mathcal{R}_{F}$ implies that $\mathcal{E}_{F} \mathcal{R}_{F}$ : $W^{1, p}(B) \rightarrow W^{1, p}(B)$ is a (continuous) projection. Furthermore, it is straightforward to verify that $\mathcal{E}_{F} \mathcal{R}_{F} \varphi=0$, iff $\mathcal{R}_{F} \varphi=0$. This implies that $\varphi \in \mathcal{W}_{F}^{1, p}(B)$, if and only if $\varphi \in W^{1, p}(B)$ and $\left(1-\mathcal{E}_{F} \mathcal{R}_{F}\right) \varphi=\varphi$. Consequently, the operator $\mathcal{P}:=1-\mathcal{E}_{F} \mathcal{R}_{F}$ is a (continuous) projection from $W^{1, p}(B)$ onto $\mathcal{W}_{F}^{1, p}(B)$.

The existence of the projector $\mathcal{P}$ allows to deduce the desired interpolation properties for the spaces $\mathcal{W}_{F}^{1, p}(\Omega)$ by purely functorial properties.

Theorem 3.5. Let $F \subset B$ be a $(d-1)$-set. Then the spaces $\mathcal{W}_{F}^{1, p}(B)(p \in] 1, \infty[)$ interpolate according to the same rules as the spaces $W^{1, p}(B)$ do. This affects any interpolation functor, in particular real and complex interpolation.

Proof. Let $\mathcal{P}$ be the projection from above. Since, for any $p \in] 1, \infty\left[, \mathcal{P}\right.$ maps $W^{1, p}(B)$ onto $\mathcal{W}_{F}^{1, p}(B)$, interpolation carries over from the spaces $W^{1, p}(B)$ to the spaces $\mathcal{W}_{F}^{1, p}(B)$ by a classical interpolation principle for complemented subspaces, see [61, Ch. 1.17.1].

In order to obtain this also for the spaces $W_{D}^{1, p}(\Omega)$, we will prove the following

Theorem 3.6. Let $F \subset \mathbb{R}^{d}$ be a $(d-1)$-set. Then the spaces $W_{F}^{1, p}\left(\mathbb{R}^{d}\right)$ and $\mathcal{W}_{F}^{1, p}\left(\mathbb{R}^{d}\right)$ in fact coincide for $p \in] 1, \infty[$. 
Proof. The inclusion $W_{F}^{1, p}\left(\mathbb{R}^{d}\right) \subseteq \mathcal{W}_{F}^{1, p}\left(\mathbb{R}^{d}\right)$ is implied by the Jonsson/Wallin result: all functions $\psi$ from $C_{F}^{\infty}\left(\mathbb{R}^{d}\right)$ vanish in a neighbourhood of $F$ and, hence, have trace 0 on $F$, i.e. $\mathcal{R}_{F} \psi=0$. Since the trace is a continuous operator into $L^{1}(F ; \rho)$, this remains true for all elements from $W_{F}^{1, p}\left(\mathbb{R}^{d}\right)$.

Conversely, assume $\psi \in \mathcal{W}_{F}^{1, p}\left(\mathbb{R}^{d}\right)$. By the definition of the projector $\mathcal{P}=1-\mathcal{E}_{F} \mathcal{R}_{F}$ one has $\mathcal{P} \psi=\psi$. Since $\psi \in \mathcal{W}_{F}^{1, p}\left(\mathbb{R}^{d}\right) \subset W^{1, p}\left(\mathbb{R}^{d}\right)$, there is a sequence $\left\{\psi_{k}\right\}_{k}$ from $C_{0}^{\infty}\left(\mathbb{R}^{d}\right)$ that converges towards $\psi$ in the $W^{1, p}\left(\mathbb{R}^{d}\right)$ topology. Clearly, then $\mathcal{P} \psi_{k} \rightarrow \mathcal{P} \psi=\psi$, and the elements $\mathcal{P} \psi_{k}$ fulfill, by the definition of $\mathcal{P}$, the condition $\mathcal{P} \psi_{k}=0$ a.e. on $F$ with respect to $\rho$. Thus we have $\mathcal{R}_{F}\left(\mathcal{P} \psi_{k}\right)=0$.

We fix $k$ and denote $\mathcal{P} \psi_{k}$ by $f$ for brevity. Our intention is to show:

$$
\text { There exists } g \in C^{\infty}\left(\mathbb{R}^{d}\right) \text { with } \operatorname{supp}(g) \cap F=\emptyset \text { and }\|f-g\|_{W^{1, p}\left(\mathbb{R}^{d}\right)} \leq \frac{1}{k} .
$$

By the construction of the projector $\mathcal{P}=1-\mathcal{E}_{F} \mathcal{R}_{F}$ and the Jonsson/Wallin results in Proposition 2.4 the function $f$ is Lipschitzian and vanishes almost everywhere on $F$. We will now show that, in fact, it vanishes identically on $F$. Let $\mathrm{x} \in F$ be an arbitrary point. Then, for every $r>0$, one has $\rho(F \cap B(\mathrm{x}, r))>0$ because $F$ is a $(d-1)$-set. Thus, in this ball there is a point $\mathrm{y} \in F$ for which $f(\mathrm{y})=0$ holds. Hence, $\mathrm{x}$ is an accumulation point of the set on which $f$ vanishes, and the claim follows from the continuity of $f$.

Let now $\left\{\zeta_{n}\right\}_{n}$ be the sequence of cut-off functions, defined on $\mathbb{R}_{+}$by

$$
\zeta_{n}(t)= \begin{cases}0, & \text { if } 0 \leq t \leq 1 / n, \\ n t-1, & \text { if } 1 / n \leq t \leq 2 / n, \\ 1, & \text { if } 2 / n<t\end{cases}
$$

Note that for $t \neq 0$ the values $\zeta_{n}(t)$ tend to 1 as $n \rightarrow \infty$. Moreover, one has $0 \leq t \zeta_{n}^{\prime}(t) \leq 2$ and $t \zeta_{n}^{\prime}(t)$ tends to 0 for all $t$. We denote by $\operatorname{dist}_{F}: \mathbb{R}^{d} \rightarrow \mathbb{R}_{+}$the function which measures the distance to the set $F$. Note that $\operatorname{dist}_{F}$ is Lipschitzian with Lipschitz constant 1 . Hence, it is a.e. differentiable with $\left|\nabla \operatorname{dist}_{F}\right| \leq 1$, see [14, Ch. 4.2.3]. Define $w_{n}:=\zeta_{n} \circ \operatorname{dist}_{F}$. Note that $w_{n} \rightarrow 1$ almost everywhere in $\mathbb{R}^{d}$ when $n \rightarrow \infty$. Moreover, since $\zeta_{n}$ is piecewise smooth, one calculates, according to the chain rule (see [20, Ch. 7.4]),

$$
\nabla w_{n}(x)= \begin{cases}\zeta_{n}^{\prime}\left(\operatorname{dist}_{F}(\mathrm{x})\right) \nabla \operatorname{dist}_{F}(\mathrm{x}), & \text { if } \left.\operatorname{dist}_{F}(\mathrm{x}) \in\right] \frac{1}{n}, \frac{2}{n}[ \\ 0, & \text { else. }\end{cases}
$$

Since $\left|\nabla \operatorname{dist}_{F}\right| \leq 1$ a.e., $\operatorname{dist}_{F} \nabla w_{n}$ is uniformly (in $n$ ) bounded a.e. and converges a.e. to 0 as $n \rightarrow \infty$. Let $f_{n}=f w_{n}$. We claim that $f_{n}-f=f\left(1-w_{n}\right) \rightarrow 0$ in $W^{1, p}\left(\mathbb{R}^{d}\right)$. By the dominated convergence theorem, $f\left(1-w_{n}\right) \rightarrow 0$ in $L^{p}\left(\mathbb{R}^{d}\right)$ since $w_{n} \rightarrow 1$. Now, for the gradient holds

$$
\nabla\left(f_{n}-f\right)=\left(1-w_{n}\right) \nabla f+f \nabla w_{n} \quad \text { a.e. on } \mathbb{R}^{d} .
$$

Again by the dominated convergence theorem, the first term converges to 0 in $L^{p}\left(\mathbb{R}^{d}\right)$. It remains to prove that $\left\|f \nabla w_{n}\right\|_{L^{p}} \rightarrow 0$. We have

$$
\left\|f \nabla w_{n}\right\|_{L^{p}}^{p}=\int_{\mathbb{R}^{d}}\left|\frac{f}{\operatorname{dist}_{F}}\right|^{p}\left|\operatorname{dist}_{F} \nabla w_{n}\right|^{p} \mathrm{dx} .
$$

Due to the fact that $f$ vanishes identically on $F$ and the Lipschitz property of $f$, the function $\frac{f}{\operatorname{dist}_{F}}$ is bounded. Hence, again dominated convergence yields $f \nabla w_{n} \rightarrow 0$ in $L^{p}\left(\mathbb{R}^{d}\right)$. The support of each function $f_{n}$ has a positive distance to the set $F$. Thus, it suffices to convolve a function $f_{n}$ (according to a sufficiently high index $n$ ) with a smooth mollifying function with small support to obtain $g$, which proves (3.3). Finally, the assertion follows from a $3 \epsilon$ argument.

Corollary 3.7. Let $B \subset \mathbb{R}^{d}$ be an open ball and $F \subset B$ be $a(d-1)$-set. Then the spaces $W_{F}^{1, p}(B)$ and $\mathcal{W}_{F}^{1, p}(B)$ in fact coincide for $\left.p \in\right] 1, \infty[$. 
Proof. The inclusion $W_{F}^{1, p}(B) \subseteq \mathcal{W}_{F}^{1, p}(B)$ is clear. Conversely, let, for any function $\psi \in \mathcal{W}_{F}^{1, p}(B)$, $\widehat{\psi}$ be a $W^{1, p}\left(\mathbb{R}^{d}\right)$-extension. Since $F$ is contained in $B$ we still have $\widehat{\psi} \in \mathcal{W}_{F}^{1, p}\left(\mathbb{R}^{d}\right)$. Hence, due to Theorem 3.6. the function $\widehat{\psi}$ may be approximated in the $W^{1, p}\left(\mathbb{R}^{d}\right)$-norm by a sequence $\left\{\psi_{k}\right\}_{k}$ from $C_{F}^{\infty}\left(\mathbb{R}^{d}\right)$. Evidently, then $\psi$ may be approximated by the sequence $\left\{\left.\psi_{k}\right|_{B}\right\}_{k}$ in the $W^{1, p}(B)$-norm.

Remark 3.8. (i) The basic idea for the proof of Theorem 3.6 is analogous to that in 36 , Prop. 3.12].

(ii) Seemingly, the coincidence of the spaces $W_{F}^{1, p}(B)$ and $\mathcal{W}_{F}^{1, p}(B)$ is only of limited, more technical interest. This, however, is not the case: on the one hand it is often considerably simpler to prove that a certain function belongs to the space $\mathcal{W}_{F}^{1, p}$, see the proof of Theorem 4.5 below, compare also [38, Ch. VIII.1] or [51, Ch. 6.6]. On the other hand, it is of course often more comfortable, if one has to prove a certain property for all elements from $\mathcal{W}_{F}^{1, p}(B)$ and may confine oneself, by density, to the functions from $C_{F}^{\infty}(B)$.

(iii) Theorem 3.6 heavily rests on the property of $F$ to be a $(d-1)$-set: suppose e.g. $p>d$ and assume that $\mathrm{x} \in F$ is an isolated point. Then, for every $\psi \in C_{F}^{\infty}(\Omega)$ one has $\psi(\mathrm{x})=0$, what clearly extends to all $\psi \in W_{F}^{1, p}(\Omega)$, since the Dirac measure $\delta_{\mathrm{x}}$ is a continuous linear form on $W^{1, p}(\Omega)$. On the other hand, the condition $\mathcal{R}_{F} \psi=0$ a.e. on $F$ does not impose a condition on $\psi$ in the point $\mathrm{x}$ because $\{\mathrm{x}\}$ is of measure 0 with respect to $\rho=\left.\mathcal{H}_{d-1}\right|_{F}$.

Corollary 3.9. Concerning real and complex interpolation, Theorem 3.5 remains true, if there $\mathcal{W}_{F}^{1, p}(B)$ is replaced by $W_{F}^{1, p}(B)$. In particular, one has for $\left.p_{0}, p_{1} \in\right] 1, \infty\left[\right.$ and $\frac{1}{p}=\frac{1-\theta}{p_{0}}+\frac{\theta}{p_{1}}$

$$
\left[W_{F}^{1, p_{0}}(B), W_{F}^{1, p_{1}}(B)\right]_{\theta}=W_{F}^{1, p}(B)=\left(W_{F}^{1, p_{0}}(B), W_{F}^{1, p_{1}}(B)\right)_{\theta, p} .
$$

Proof. The assertion concerning complex interpolation is immediate from Theorem 3.5 and Theorem 3.6. which also imply the right equality. Considering real interpolation, one gets

$$
\left(W_{F}^{1, p_{0}}(B), W_{F}^{1, p_{1}}(B)\right)_{\theta, q}=\left(\mathcal{W}_{F}^{1, p_{0}}(B), \mathcal{W}_{F}^{1, p_{1}}(B)\right)_{\theta, q}
$$

According to Theorem 3.5, the right hand side is some Besov space (see [61, Ch. 2.4.2]) including again the trace-zero condition on $F$. It is clear that $C_{F}^{\infty}(B)$ is contained in this space. What remains to show is that $C_{F}^{\infty}(B)$ is also dense in this space.

Let us suppose, without loss of generality, $p_{1}>p_{0}$. By definition, $C_{F}^{\infty}(B)$ is dense in $W_{F}^{1, p_{1}}(B)$ with respect to its natural topology. Moreover, $W_{F}^{1, p_{1}}(B)$ is dense in the interpolation space (3.5) (see [61, Ch. 1.6.2]), and the topology of this interpolation space is weaker than that of $W_{F}^{1, p_{1}}(B)$. Hence, $C_{F}^{\infty}(B)$ is indeed dense in the corresponding interpolation space, or, in other words: the interpolation space is the closure of $C_{F}^{\infty}(B)$ with respect to the corresponding Besov topology.

Remark 3.10. Concerning real interpolation, the interpolation indices $(\theta, \infty)$ have indeed to be excluded, compare [61, Ch. 1.6.2]. The crucial point is that the smaller space has to remain dense in the corresponding interpolation space.

We now turn to the proof of Theorem 3.3. We first introduce the following definition.

Definition 3.11. We denote by $\mathfrak{R}: W^{1, p}(B) \rightarrow W^{1, p}(\Omega)$ the canonical restriction operator.

Remark 3.12. It is not hard to see that the canonical restriction operator $\mathfrak{R}: W^{1, p}(B) \rightarrow$ $W^{1, p}(\Omega)$ gives rise to a restriction operator $\mathfrak{R}: W_{D}^{1, p}(B) \rightarrow W_{D}^{1, p}(\Omega)$ - for which we also maintain the notation $\mathfrak{R}$. Note that $\mathfrak{E}$ and $\mathfrak{R}$ are consistent on the sets $\left\{W_{D}^{1, p}(B)\right\}_{p \in[1, \infty[}$ and $\left\{W_{D}^{1, p}(\Omega)\right\}_{p \in[1, \infty[}:$ if $q>p$, then $\mathfrak{R}: W_{D}^{1, q}(B) \rightarrow W_{D}^{1, q}(\Omega)$ is the restriction of $\mathfrak{R}: W_{D}^{1, p}(B) \rightarrow$ $W_{D}^{1, p}(\Omega)$ and $\mathfrak{E}: W_{D}^{1, q}(\Omega) \rightarrow W_{D}^{1, q}(B)$ is the restriction of $\mathfrak{E}: W_{D}^{1, p}(\Omega) \rightarrow W_{D}^{1, p}(B)$. 
Finally, one observes that, for every $p \in\left[1, \infty\left[\right.\right.$, the operators $\mathfrak{R}: W_{D}^{1, p}(B) \rightarrow W_{D}^{1, p}(\Omega)$ and $\mathfrak{E}: W_{D}^{1, p}(\Omega) \rightarrow W_{D}^{1, p}(B)$ form a retraction/coretraction pair, see [61, Ch. 1.2.4].

Proof of Theorem 3.3. Let $B \supset \bar{\Omega}$ be the ball introduced above. Firstly, Corollary 3.9 shows how the spaces from the family $\left\{W_{D}^{1, p}(B)\right\}_{p \in] 1, \infty[}$ interpolate. Secondly, the extension/restriction operators $\mathfrak{E} / \mathfrak{R}$ (compare Remark 3.2 (ii) together with the retraction/coretraction theorem, see [61, Ch. 1.2.4], allow to carry over interpolation between spaces from $\left\{W_{D}^{1, p}(B)\right\}_{p \in] 1, \infty[}$ to the spaces from $\left\{W_{D}^{1, p}(\Omega)\right\}_{p \in] 1, \infty[}$.

\section{The EXTENSION OPERATOR}

As already mentioned in Remark 3.2 , the condition of the extendability for $W_{D}^{1, p}(\Omega)$ within the same class is an abstract one which should be supported by geometric conditions on $\Omega$ and on $D$. We will do this within this section. In a first step we will establish three general principles. First, we open the possibility of passing from the domain $\Omega$ to another domain $\Omega_{\bullet}$ with a reduced Dirichlet boundary part, while $\Gamma=\partial \Omega \backslash D$ remains part of $\partial \Omega_{\bullet}$. In most cases this improves the boundary geometry in view of the $W^{1, p}$-extendability, see the example in Figure 2 above. Secondly, we show that only the local geometry of the domain around the boundary part $\Gamma$ plays a role for the existence of such an extension operator. Thirdly, we prove that - under very general geometric assumptions - the extended functions do have the adequate trace behavior on $D$ for every extension operator.

In the second subsection we then give conditions for geometries around the boundary part $\Gamma$, which do - together with the results from Subsection 4.1 - really imply the validity of Assumption 3.1 .

4.1. Sobolev extension: general features. The first point we address is the following: as in Figure 2 there may be boundary parts which carry a Dirichlet condition and belong to the inner of the closure of the domain under consideration. Then one can extend the functions on $\Omega$ by 0 to such boundary part, thereby enlarging the domain and simplifying the boundary geometry. In the following we make this precise.

Lemma 4.1. Let $\Omega \subset \mathbb{R}^{d}$ be a domain and let $E \subset \partial \Omega$ be compact. Define $\Omega$. as the interior of the set $\Omega \cup E$. Then the following holds true.

(i) The set $\Omega_{\bullet}$ is again a domain, $\Xi:=\partial \Omega \backslash E$ is a (relatively) open subset of $\partial \Omega$. and $\partial \Omega_{\bullet}=\Xi \cup\left(E \cap \partial \Omega_{\bullet}\right)$.

(ii) Extending functions from $W_{E}^{1, p}(\Omega)$ by 0 to $\Omega_{\bullet}$, one obtains an isometric extension operator $\operatorname{Ext}\left(\Omega, \Omega_{\bullet}\right)$ from $W_{E}^{1, p}(\Omega)$ onto $W_{E}^{1, p}\left(\Omega_{\bullet}\right)$.

Proof. $\quad$ (i) Due to the connectednes of $\Omega$ and the set inclusion $\Omega \subset \Omega_{\bullet} \subset \bar{\Omega}$, the set $\Omega_{\bullet}$ is also connected, and, hence, a domain. Obviously, one has $\overline{\Omega_{\bullet}}=\bar{\Omega}$. This, together with the inclusion $\Omega \subset \Omega_{\bullet}$ leads to $\partial \Omega_{\bullet} \subset \partial \Omega$. Since $\Xi \cap \Omega_{\bullet}=\emptyset$, one gets $\Xi \subset \partial \Omega_{\bullet}$. Furthermore, $\Xi$ was relatively open in $\partial \Omega$, the more it is relatively open in $\partial \Omega_{\bullet}$.

The last asserted equality follows from $\partial \Omega_{\bullet}=\left(\Xi \cap \partial \Omega_{\bullet}\right) \cup\left(E \cap \partial \Omega_{\bullet}\right)$ and $\Xi \subset \partial \Omega_{\bullet}$.

(ii) Consider any $\psi \in C_{E}^{\infty}\left(\mathbb{R}^{d}\right)$ and its restriction $\left.\psi\right|_{\Omega}$ to $\Omega$. Since the support of $\psi$ has a positive distance to $E$, one may extend $\left.\psi\right|_{\Omega}$ by 0 to the whole of $\Omega$ • without destroying the $C^{\infty}$-property. Thus, this extension operator provides a linear isometry from $C_{E}^{\infty}(\Omega)$ onto $C_{E}^{\infty}\left(\Omega_{\bullet}\right)$ (if both are equipped with the $W^{1, p}$-norm). This extends to a linear extension operator $\operatorname{Ext}\left(\Omega, \Omega_{\bullet}\right)$ from $W_{E}^{1, p}(\Omega)$ onto $W_{E}^{1, p}\left(\Omega_{\bullet}\right)$, see the two following commutative diagrams: 

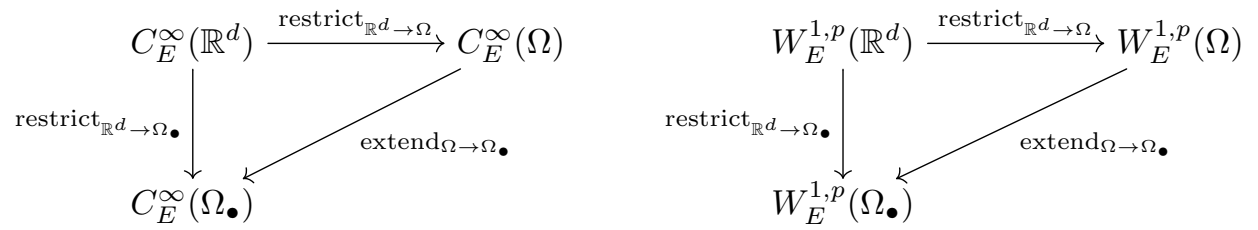

Remark 4.2. $\quad$ (i) The reader should notice that no assumptions on $E$ beside compactness are necessary.

(ii) Observe that, after having extended the functions, being defined on $\Omega$, to $\Omega_{\bullet}$, the 'Dirichlet crack' $\Sigma$ in Figure 2 has vanished, and one ends up with the whole cube. Here the problem of extending Sobolev functions is almost trivial. We suppose that this is the generic case - at least for applied problems.

The above considerations suggest the following procedure: extend the functions from $W_{E}^{1, p}(\Omega)$ first to $\Omega_{\bullet}$, and afterwards to the whole of $\mathbb{R}^{d}$. The next lemma will show that this approach is universal.

Lemma 4.3. Every linear, continuous extension operator $\mathfrak{F}: W_{E}^{1, p}(\Omega) \rightarrow W_{E}^{1, p}\left(\mathbb{R}^{d}\right)$ factorizes in the following manner: there is a linear, continuous extension operator $\mathfrak{F}_{\bullet}: W_{E}^{1, p}\left(\Omega_{\bullet}\right) \rightarrow W_{E}^{1, p}\left(\mathbb{R}^{d}\right)$, such that $\mathfrak{F}=\mathfrak{F} \bullet \operatorname{Ext}\left(\Omega, \Omega_{\bullet}\right)$.

Proof. Let $\mathfrak{S}$ be the restriction operator from $W_{E}^{1, p}\left(\Omega_{\bullet}\right)$ to $W_{E}^{1, p}(\Omega)$. Then we define, for every $f \in W_{E}^{1, p}\left(\Omega_{\bullet}\right), \mathfrak{F}_{\bullet} f:=\mathfrak{F} \mathfrak{S} f$. We obtain $\mathfrak{F} \bullet \operatorname{Ext}\left(\Omega, \Omega_{\bullet}\right)=\mathfrak{F} \mathfrak{S} \operatorname{Ext}\left(\Omega, \Omega_{\bullet}\right)=\mathfrak{F}$. This shows that the factorization holds algebraically. But one also has

$$
\begin{aligned}
\left\|\mathfrak{F}_{\bullet} \operatorname{Ext}\left(\Omega, \Omega_{\bullet}\right) f\right\|_{W_{E}^{1, p}\left(\mathbb{R}^{d}\right)} & =\|\mathfrak{F} f\|_{W_{E}^{1, p}\left(\mathbb{R}^{d}\right)} \leq\|\mathfrak{F}\|_{\mathcal{L}\left(W_{E}^{1, p}(\Omega) ; W_{E}^{1, p}\left(\mathbb{R}^{d}\right)\right)}\|f\|_{W_{E}^{1, p}(\Omega)} \\
& =\|\mathfrak{F}\|_{\mathcal{L}\left(W_{E}^{1, p}(\Omega) ; W_{E}^{1, p}\left(\mathbb{R}^{d}\right)\right)}\left\|\operatorname{Ext}\left(\Omega, \Omega_{\bullet}\right) f\right\|_{W_{E}^{1, p}\left(\Omega_{\bullet}\right)} .
\end{aligned}
$$

Having extended the functions already to $\Omega_{\bullet}$, one may proceed as follows: $E$ was compact, thus $E_{\bullet}:=E \cap \partial \Omega_{\bullet}$ is closed in $\partial \Omega_{\bullet}$. So one can now consider the space $W_{E_{\bullet}}^{1, p}\left(\Omega_{\bullet}\right)$ and has then the task to establish an extension operator for this space - while afterwards taking into account that the original functions were 0 also on the set $E \cap \Omega_{\bullet}$.

Definition 4.4. Let $\Lambda \subset \mathbb{R}^{d}$ be a bounded domain and suppose $p \in[1, \infty[$. Then we say that $\Lambda$ is a $W^{1, p}$-extension domain, if there exists a linear, continuous extension operator $\mathfrak{F}_{p}$ : $W^{1, p}(\Lambda) \rightarrow W^{1, p}\left(\mathbb{R}^{d}\right)$. We call $\Lambda$ a Sobolev extension domain, if it is a $W^{1,1}$-extension domain and the restrictions of $\mathfrak{F}_{1}$ give continuous extension operators $\mathfrak{F}_{p}: W^{1, p}(\Lambda) \rightarrow W^{1, p}\left(\mathbb{R}^{d}\right)$ for any $p \in] 1, \infty[$.

We now come to the second aim of this subsection, that is to show that a local extension property of $\Lambda$ around points of $\overline{\partial \Lambda \backslash F}$ already gives an extension operator for the space $W_{F}^{1, p}(\Lambda)$. Here one should think of $\Lambda$ as being either $\Omega$ or $\Omega_{\bullet}$.

Theorem 4.5. Fix $p \in[1, \infty[$. Let $\Lambda$ be a bounded domain and let $F$ be a closed part of its boundary. Assume that, for every $\mathrm{x} \in \overline{\partial \Lambda \backslash F}$, there is an open neighbourhood $U_{\mathrm{x}}$ of $\mathrm{x}$ such that $\Lambda \cap U_{\mathrm{x}}$ is a $W^{1, p}$-extension domain. Then there is a continuous extension operator $\mathfrak{F}_{p}$ : $W_{F}^{1, p}(\Lambda) \rightarrow W^{1, p}\left(\mathbb{R}^{d}\right)$.

Proof. For every $\mathrm{x} \in \overline{\partial \Lambda \backslash F}$, let $U_{\mathrm{x}}$ be the open neighbourhood of $\mathrm{x}$ from the assumption. Let $U_{\mathrm{x}_{1}}, \ldots, U_{\mathrm{x}_{n}}$ be a finite subcovering of $\overline{\partial \Lambda \backslash F}$. Since the compact set $\overline{\partial \Lambda \backslash F}$ is contained in the open set $\bigcup_{j} U_{\mathrm{x}_{j}}$, there is an $\epsilon>0$, such that the sets $U_{\mathrm{x}_{1}}, \ldots, U_{\mathrm{x}_{n}}$, together with the open 
set $U:=\{\mathrm{y} \in \Omega: \operatorname{dist}(\mathrm{y}, \overline{\partial \Lambda \backslash F})>\epsilon\}$, form an open covering of $\bar{\Lambda}$. Hence, on $\bar{\Lambda}$ there is a $C^{\infty}$-partition of unity $\eta, \eta_{1}, \ldots, \eta_{n}$, with the properties $\operatorname{supp}(\eta) \subset U, \operatorname{supp}\left(\eta_{j}\right) \subset U_{\mathrm{x}_{j}}$.

Assume $\psi \in C_{F}^{\infty}(\Lambda)$. Then $\eta \psi \in C_{0}^{\infty}(\Lambda) \subset W_{0}^{1, p}(\Lambda)$. If one extends this function by 0 outside of $\Lambda$, then one obtains a function $\varphi \in C_{\partial \Lambda}^{\infty}\left(\mathbb{R}^{d}\right) \subset C_{F}^{\infty}\left(\mathbb{R}^{d}\right) \subset W_{F}^{1, p}\left(\mathbb{R}^{d}\right)$ with the property $\|\varphi\|_{W^{1, p}\left(\mathbb{R}^{d}\right)}=\|\eta \psi\|_{W^{1, p}(\Lambda)}$.

Now, for every fixed $j \in\{1, \ldots, n\}$, consider the function $\psi_{j}:=\eta_{j} \psi \in W^{1, p}\left(\Lambda \cap U_{\mathbf{x}_{j}}\right)$. Since $\Lambda \cap U_{\mathrm{x}_{j}}$ is a $W^{1, p}$-extension domain by supposition, there is an extension of $\psi_{j}$ to a $W^{1, p}\left(\mathbb{R}^{d}\right)$ function $\varphi_{j}$ together with an estimate $\left\|\varphi_{j}\right\|_{W^{1, p}\left(\mathbb{R}^{d}\right)} \leq c\left\|\psi_{j}\right\|_{W^{1, p}\left(\Lambda \cap U_{\mathrm{x}_{j}}\right)}$, where $c$ is independent from $\psi$. Clearly, one has a priori no control on the behaviour of $\varphi_{j}$ on the set $\Lambda \backslash U_{\mathbf{x}_{j}}$. In particular $\varphi_{j}$ may there be nonzero and, hence, cannot be expected to coincide with $\eta_{j} \psi$ on the whole of $\Lambda$. In order to correct this, let $\zeta_{j}$ be a $C_{0}^{\infty}\left(\mathbb{R}^{d}\right)$-function which is identically 1 on $\operatorname{supp}\left(\eta_{j}\right)$ and has its support in $U_{\mathrm{x}_{j}}$. Then $\eta_{j} \psi$ equals $\zeta_{j} \varphi_{j}$ on all of $\Lambda$. Consequently, $\zeta_{j} \varphi_{j}$ really is an extension of $\eta_{j} \psi$ to the whole of $\mathbb{R}^{d}$ which, additionally, satisfies the estimate

$$
\left\|\zeta_{j} \varphi_{j}\right\|_{W^{1, p}\left(\mathbb{R}^{d}\right)} \leq c\left\|\varphi_{j}\right\|_{W^{1, p}\left(\mathbb{R}^{d}\right)} \leq c\left\|\eta_{j} \psi\right\|_{W^{1, p}\left(\Lambda \cap U_{\mathbf{x}_{j}}\right)} \leq c\|\psi\|_{W^{1, p}(\Lambda)},
$$

where $c$ is independent from $\psi$. Thus, defining $\mathfrak{F}_{p}(\psi)=\varphi+\sum_{j} \zeta_{j} \varphi_{j}$ one gets a linear, continuous extension operator $\mathfrak{F}_{p}$ from $C_{F}^{\infty}(\Lambda)$ into $W^{1, p}\left(\mathbb{R}^{d}\right)$. By density, $\mathfrak{F}_{p}$ extends uniquely to a linear, continuous operator

$$
\mathfrak{F}_{p}: W_{F}^{1, p}(\Lambda) \rightarrow W^{1, p}\left(\mathbb{R}^{d}\right) .
$$

Remark 4.6. (i) Observe that the set $F=\partial \Omega_{\bullet} \cap E$ is not necessarily again a $(d-1)$-set; possibly one even has $\mathcal{H}_{d-1}(F)=0$. (Take Figure 2 and suppose that this time only $\Sigma$ forms the whole Dirichlet part of the boundary.) The reader should carefully notice, that this does not affect the considerations in Theorem 4.5

(ii) Of course, one gets uniformity with respect to $p$ from any subinterval of $[1, \infty[$ if one invests uniformity concerning the extension property for the local domains $\Lambda \cap U_{\mathrm{x}}$.

(iii) If one aims at an extension operator $\mathfrak{E}: W_{D}^{1, p}(\Omega) \rightarrow W_{D}^{1, p}\left(\mathbb{R}^{d}\right)$, one is free to modify the domain $\Omega$ to $\Omega_{\bullet}$ - or not. In most cases the local geometry improves (concerning Sobolev extension), but we are unable to show that this is always the case - irrespective of Lemma 4.3. On the other hand, we have no examples where the situation becomes worse.

Theorem 4.5 yields a Sobolev extension operator from $W_{F}^{1, p}(\Lambda)$ to $W^{1, p}\left(\mathbb{R}^{d}\right)$. However, our aim is to show that it does not destroy the boundary behavior, which means that it even maps to $W_{F}^{1, p}\left(\mathbb{R}^{d}\right)$. We will now turn to this question.

Lemma 4.7. Suppose that $\Lambda \subset \mathbb{R}^{d}$ is a domain and $F \subseteq \partial \Lambda$ is a (closed) (d-1)-set. Moreover, assume that for $\mathcal{H}_{d-1}$-almost all points $\mathrm{y} \in F$, balls around $\mathrm{y}$ in $\Lambda$ have asymptotically nonvanishing relative volume, i.e.

$$
\liminf _{r \mapsto 0} \frac{\mid B(\mathrm{y} ; r) \cap \Lambda) \mid}{r^{d}}>0 .
$$

Let $\psi \in C_{F}^{\infty}(\Lambda)$ and $\left.p \in\right] 1, \infty\left[\right.$. If there is an extension $\widehat{\psi} \in W^{1, p}\left(\mathbb{R}^{d}\right)$ of $\psi$, then $\widehat{\psi} \in W_{F}^{1, p}\left(\mathbb{R}^{d}\right)$.

Proof. One first proves the property $\widehat{\psi} \in \mathcal{W}_{F}^{1, p}\left(\mathbb{R}^{d}\right)$. In fact, $\operatorname{since} \operatorname{supp}(\psi)$ has a positive distance to $F$, one clearly has $\lim _{r \rightarrow 0} \frac{1}{|\Lambda \cap B(\mathrm{y}, r)|} \int_{\Lambda \cap B(\mathrm{y}, r)} \psi(\mathrm{x}) d \mathrm{x}=0$ for all $\mathrm{y} \in F$. From this, one deduces

$$
\lim _{r \rightarrow 0} \frac{1}{|B(\mathrm{y}, r)|} \int_{B(\mathrm{y}, r)} \widehat{\psi}(\mathrm{x}) d \mathrm{x}=0 \quad \text { for } \quad \mathcal{H}_{d-1} \text {-almost all } \mathrm{y} \in F .
$$

The proof of this runs along the same lines as the proof of [38, Ch. VIII Prop. 2]; with two differences: 
Firstly, one has to take the measure $\mu$ here as $\left.\mathcal{H}_{d-1}\right|_{F}$ instead of $\left.\mathcal{H}_{d-1}\right|_{\partial \Lambda}$ there. In order to do so, one has to show that this measure has the required functional analytic quality - and this is the case.

Secondly, one observes that the liminf in 4.2 does in fact not need not to have a uniform lower bound for ( $\mathcal{H}_{d-1}$-almost) all $\mathrm{y} \in F$, the above condition suffices.

But (4.3) implies $\widehat{\psi} \in \mathcal{W}_{F}^{1, p}\left(\mathbb{R}^{d}\right)$, recall Remark 2.6. Having this at hand, one applies Theorem 3.6 .

Corollary 4.8. Assume that there is a linear, continuous extension operator $\mathfrak{E}: W_{D}^{1, p}(\Omega) \rightarrow$ $W^{1, p}\left(\mathbb{R}^{d}\right)$. Then, under the assumptions of Lemma 4.7. E maps into the space $W_{D}^{1, p}\left(\mathbb{R}^{d}\right)$.

In the case where an extension operator from $W_{F}^{1, p}(\Omega)$ into $W^{1, p}\left(\mathbb{R}^{d}\right)$ operates uniformly in $p$, things become much simpler - as the following result shows:

Lemma 4.9. Let $\Lambda$ be a bounded domain, and let $F \subset \partial \Lambda$ be a (closed) (d-1)-set. If there is continuous extension operator $\mathfrak{E}: W_{F}^{1,1}(\Lambda) \rightarrow W_{F}^{1,1}\left(\mathbb{R}^{d}\right)$ which acts as a continuous operator $\mathfrak{E}: W_{F}^{1, p}(\Lambda) \rightarrow W^{1, p}\left(\mathbb{R}^{d}\right)$ for all $\left.\left.p \in\right] 1, d+\epsilon\right]$ (d being the space dimension and $\epsilon>0$ ). Then, for every $p \in] 1, d+\epsilon]$, E maps the space $W_{F}^{1, p}(\Lambda)$ even into $W_{F}^{1, p}\left(\mathbb{R}^{d}\right)$.

Proof. Fix $p \in] 1, d+\epsilon]$ and assume first $\psi \in C_{F}^{\infty}(\Lambda)$. Then the extension $\mathfrak{E} \psi$ does not belong only to $W^{1, p}\left(\mathbb{R}^{d}\right)$ but even to $W^{1, d+\epsilon}\left(\mathbb{R}^{d}\right)$. Hence, $\mathfrak{E} \psi$ has a representative which is Hölder continuous. Moreover, it is clear that this representative is identical 0 on $F$. This leads to the property $\mathfrak{E} \psi \in \mathcal{W}_{F}^{1, p}\left(\mathbb{R}^{d}\right)$, according to the $(d-1)$-property of $F$, cf. Remark 2.6 . But, thanks to Theorem 3.6. the spaces $\mathcal{W}_{F}^{1, p}\left(\mathbb{R}^{d}\right)$ and $W_{F}^{1, p}\left(\mathbb{R}^{d}\right)$ coincide - what implies $\mathfrak{E} \psi \in W_{F}^{1, p}\left(\mathbb{R}^{d}\right)$. This proves the assertion for all elements from the dense subspace $C_{F}^{\infty}(\Lambda)$; what implies the claim by the continuity of $\mathfrak{E}$.

4.2. Geometric conditions. In this subsection we will present geometric conditions on the boundary part $\overline{\partial \Lambda \backslash F}$, such that the local sets $\Lambda \cap U_{\mathrm{x}_{j}}$ really admit the Sobolev extension property required in Theorem 4.5. A first condition, completely sufficient for the treatment of most real world problems, is the following:

Assumption 4.10. Let $\Omega$ and $\Gamma$ be as in Assumption 2.2 and let $\Lambda$ be either $\Omega$ or $\Omega_{\bullet}$, cf. Remark 4.6 (iii). For every $\mathrm{x} \in \bar{\Gamma}$ there is an open neighbourhood $U_{\mathrm{x}}$ of $\mathrm{x}$ and a bi-Lipschitz mapping $\phi_{\mathrm{x}}$ from $U_{\mathrm{x}}$ onto a cube, such that $\phi_{\mathrm{x}}\left(\Lambda \cap U_{\mathrm{x}}\right)$ is the (lower) half cube and $\partial \Lambda \cap U_{\mathrm{x}}$ is mapped onto the top surface of the half cube.

A proof for the fact that this condition really leads to the required extension operator is given in [13] for the case $p=2$. It carries over, however, to $p \in[1, \infty[-$ word by word.

Another relevant condition that assures the existence of a Sobolev extension operator is that of Jones [37. In order to formulate this we need the following definition.

Definition 4.11. Let $\Upsilon \subset \mathbb{R}^{d}$ be a domain and $\varepsilon, \delta>0$. Assume that any two points $\mathrm{x}, \mathrm{y} \in \Upsilon$, with distance not larger than $\delta$, can be connected within $\Upsilon$ by a rectifiable $\operatorname{arc} \gamma$ with lenght $l(\gamma)$, such that the following two conditions are satisfied for all points $\mathrm{z}$ from the curve $\gamma$ :

$$
l(\gamma) \leq \frac{1}{\varepsilon}\|\mathrm{x}-\mathrm{y}\|, \quad \text { and } \quad \frac{\|\mathrm{x}-\mathrm{z}\|\|\mathrm{y}-\mathrm{z}\|}{\|\mathrm{x}-\mathrm{y}\|} \leq \frac{1}{\varepsilon} \operatorname{dist}\left(\mathrm{z}, \Upsilon^{c}\right) .
$$

Then $\Upsilon$ is called $(\varepsilon, \delta)$-domain in the spirit of Jones.

Proposition 4.12. If $\Upsilon$ is an $(\varepsilon, \delta)$-domain, then it is a Sobolev extension domain.

Remark 4.13. This famous result is due to Jones [37]. Bounded $(\varepsilon, \delta)$-domains are known to be uniform domains, see [62, Ch. 4.2], compare also [37, [49, [50, [48, for further information. 
Although the uniformness property is not necessary for a domain to be a Sobolev extension domain (see [64]) it seems presently to be the broadest class of domains for which this extension property holds - at least if one aims at all $p \in] 1 \infty[$. E.g. it contains Koch's snowflake, cf. 37.

In view of these considerations, we can formulate the following criteria for the existence of the required extension operator. cf. Theorems 4.5 and Lemma 4.9 .

Theorem 4.14. Let $\Omega, \Gamma$ and $D$ be as in Assumption 2.2 and let $\Lambda$ be either $\Omega$ or $\Omega_{\bullet}$, cf. Remark 4.6 (iii). Suppose that Assumption 4.10 is fullfilled or suppose that for every $\mathrm{x} \in \bar{\Gamma}$ there is an open, bounded neighbourhood $U_{\mathrm{x}}$ of $\mathrm{x}$, such that $U_{\mathrm{x}} \cap \Lambda$ is an $(\varepsilon, \delta)$-domain. Then there exists a continuous, linear extension operator $\mathfrak{E}: W_{D}^{1, p}(\Omega) \rightarrow W_{D}^{1, p}\left(\mathbb{R}^{d}\right)$.

Proof. Both geometric configurations admit a continuous extension operator $W_{D}^{1, p}(\Omega) \rightarrow W^{1, p}\left(\mathbb{R}^{d}\right)$, according Theorem 4.5 - which is even uniform in $p \in] 1, \infty[$. Thus, Lemma 4.9 applies.

Remark 4.15. Lemma 4.9 applied to the special case of Jones' extension operator, provides an alternative proof of [4, Theorem 1.3] in case of first order Sobolev spaces, if $D$ is a $(d-1)$-set. In [4] this is achieved, even for arbitrary compact boundary parts $D$, by a deep analysis of the support properties of the functions obtained by Jones' extension operator.

\section{Elliptic AND PARABolic Regularity}

In this section we prove that the interpolation property of the spaces $W_{D}^{1, p}(\Omega)$ - in conjuction with a famous result of Sneiberg [59] - already leads to substantial regularity results within this scale of spaces.

5.1. Isomorphism property for elliptic operators. In this subsection we will prove the announced elliptic regularity theorem, which we consider as the second essential result of this work. Let us emphasize that spaces like $W_{D}^{-1, p}$ are adequate for the treatment of elliptic/parabolic equations, if the right hand side possibly contains distributional objects like surface densities. In electrostatics, for instance, a charge density on an interface causes a jump in the normal component of the dielectric displacement, see for instance [60, Chapter 1].

Let us first recall the definition of a scale of Banach spaces (see [45, Ch.1], compare also [61, Ch. 1.19.4]).

Definition 5.1. Consider a closed interval $I \subset[0, \infty[$ and a family of complex Banach spaces $\left\{X_{\tau}\right\}_{\tau \in I}$. One calls this family a (complex) scale (of Banach spaces), if

(i) $X_{\beta}$ embeds continuously and densely in $X_{\alpha}$, whenever $\beta>\alpha$.

(ii) For every triple $\alpha, \beta, \gamma \in I$ satisfying $\alpha<\beta<\gamma$ there is a positive constant $c(\alpha, \beta, \gamma)$ such that for all $\psi \in X_{\gamma}$ the following interpolation inequality holds

$$
\|\psi\|_{X_{\beta}} \leq c(\alpha, \beta, \gamma)\|\psi\|_{X_{\alpha}}^{\frac{\gamma-\beta}{\gamma-\alpha}}\|\psi\|_{X_{\gamma}}^{\frac{\beta-\alpha}{\gamma-\alpha}}
$$

We associate to the families $\left\{W_{D}^{1, p}(\Omega)\right\}_{p \in] 1, \infty[}$ and $\left\{W_{D}^{-1, p}(\Omega)\right\}_{p \in] 1, \infty[}$ Banach scales in the following manner

Definition 5.2. For $\tau \in] 0,1\left[\right.$ we define $X_{\tau}:=W_{D}^{1,(1-\tau)^{-1}}(\Omega)$ and $Y_{\tau}:=W_{D}^{-1,(1-\tau)^{-1}}(\Omega)$.

Lemma 5.3. Let Assumptions 2.2 and 3.1 be satisfied. Then, for all $\left.\tau_{1}, \tau_{2} \in\right] 0,1\left[\right.$ with $\tau_{1}<\tau_{2}$, the families $\left\{X_{\tau}\right\}_{\tau \in\left[\tau_{1}, \tau_{2}\right]}$ and $\left\{\bar{Y}_{\tau}\right\}_{\tau \in\left[\tau_{1}, \tau_{2}\right]}$ form Banach scales.

Proof. We show more, namely: for every $\alpha, \beta, \gamma \in] 0,1[$ with $\alpha<\beta<\gamma$ one has

$$
X_{\beta}=\left[X_{\alpha}, X_{\gamma}\right]_{\frac{\beta-\alpha}{\gamma-\alpha}} \text { and } Y_{\beta}=\left[Y_{\alpha}, Y_{\gamma}\right]_{\frac{\beta-\alpha}{\gamma-\alpha}} \text {. }
$$


Putting $\theta:=\frac{\beta-\alpha}{\gamma-\alpha}$, one has $1-\beta=(1-\alpha)(1-\theta)+(1-\gamma) \theta$. Thus the equalities in $(5.2$ follow from Theorem 3.3 and Corollary 3.4 . The inequality $(5.1)$ is then the interpolation inequality for complex interpolation.

Throughout the rest of the paper we assume that the following is satisfied:

Assumption 5.4. Let $\mu$ be a matrix valued, bounded, measurable, elliptic function on $\Omega$. The latter condition means that $\operatorname{Re}(\mu(\mathrm{x}) \xi \cdot \bar{\xi}) \geq \mu_{\bullet}|\xi|^{2}$ for some positive constant $\mu_{\bullet}$, all $\xi \in \mathbb{C}^{d}$ and almost all $\mathrm{x} \in \Omega$.

Definition 5.5. For every $p \in] 1, \infty\left[\right.$, we define the operator $-\nabla \cdot \mu \nabla: W_{D}^{1, p}(\Omega) \rightarrow W_{D}^{-1, p}(\Omega)$ by

$$
\langle-\nabla \cdot \mu \nabla v, w\rangle:=\int_{\Omega} \mu \nabla v \cdot \nabla \bar{w} d \mathrm{x}, \quad v \in W_{D}^{1, p}(\Omega), w \in W_{D}^{1, p^{\prime}}(\Omega),
$$

where $\langle\cdot, \cdot\rangle$ denotes the antidual pairing between $\left(W_{D}^{1, p^{\prime}}(\Omega)\right)^{\prime}=W_{D}^{-1, p}(\Omega)$ and $W_{D}^{1, p^{\prime}}(\Omega)$.

Let us briefly recall the (well known) connection between the operator $-\nabla \cdot \mu \nabla: W_{D}^{1, p}(\Omega) \rightarrow$ $W_{D}^{-1, p}(\Omega)$ and mixed boundary value problems. For this, consider the mixed boundary value problem

$$
\begin{aligned}
-\nabla \cdot(\mu \nabla u) & =f_{\Omega} \in L^{p}(\Omega) \\
\left.u\right|_{D} & =0 \\
\nu \cdot \mu \nabla u & =f_{\Gamma} \in L^{p}(\Gamma),
\end{aligned}
$$

where $\nu$ denotes the outward unit normal of the domain. Here (5.4) is to be understood in the sense of distributions on $\Omega$ and $\sqrt{5.6}$ is to be understood in a generalized sense. If one defines $f \in W_{D}^{-1, p}(\Omega)$ by $\langle f, v\rangle:=\int_{\Omega} f_{\Omega} \bar{v} d \mathrm{x}+\int_{\Gamma} f_{\Gamma} \bar{v} d \mathcal{H}_{d-1}$, then, under reasonable assumptions on $\Omega, \Gamma$ and $D$ an adequate functional analytic formulation of the problem $5.4-5.6$ is the operator equation $-\nabla \cdot \mu \nabla u=f$, see [5, Ch. 1.2], [17, Ch II.2]) or [7] for details; compare also [34] and [12. for a different approach.

When restricting the range space of the operator $-\nabla \cdot \mu \nabla$ to $L^{2}(\Omega)$, one obtains an operator for which the elements $\psi$ of its domain satisfy the conditions $\left.\psi\right|_{D}=0$ in the sense of traces and $\nu \cdot \mu \nabla \psi=0$ on $\Gamma$ in a generalized sense.

It follows the second main result of this work:

Theorem 5.6. Let Assumptions 2.2, 3.1 and 5.4 be satisfied. Then there is an open interval I containing 2 , such that the operator

$$
-\nabla \cdot \mu \nabla+1: W_{D}^{1, p}(\Omega) \rightarrow W_{D}^{-1, p}(\Omega)
$$

is a topological isomorphism for all $p \in I$.

Proof. We know from Lemma 5.3 that the families $\left\{X_{\tau}\right\}_{\tau \in[\alpha, \beta]}$ and $\left\{Y_{\tau}\right\}_{\tau \in[\alpha, \beta]}$ with $\left.\alpha, \beta \in\right] 0,1[$ form Banach scales. The mapping in (5.7) is continuous for all $p$, due to the boundedness of the coefficient function $\mu$, what is to be interpreted as the continuity of

$$
-\nabla \cdot \mu \nabla+1: X_{\tau} \rightarrow Y_{\tau}
$$

for all $\tau \in] 0,1\left[\right.$. Lastly, the quadratic form $W_{D}^{1,2}(\Omega) \ni \psi \mapsto \int_{\Omega}\left(\mu \nabla \psi \cdot \nabla \bar{\psi}+|\psi|^{2}\right) d \mathrm{x}$ is coercive. Hence the Lax-Milgram lemma gives the continuity of the inverse of (5.7) in the case of $p=2$. In the scale terminology, this is nothing else but the continuity of $(-\nabla \cdot \mu \nabla+1)^{-1}: Y_{\tau} \rightarrow X_{\tau}$ in case of $\tau=\frac{1}{2}$. A deep theorem of Sneiberg ([59], see also [3, Lemma 4.16] or [63]) says that the set of parameters $\tau$ for which 5 is a topological isomorphism, is open. Since $\frac{1}{2}$ is contained in this set, it cannot be empty. 
Remark 5.7. $\quad$ (i) Again interpolation shows that the values $p$, for which (5.7) is a topological isomorphism, form an interval $I$. Due to the Sneiberg result, this interval is an open one.

(ii) If $\mu$ takes real, symmetric matrices as values, then

$$
-\nabla \cdot \mu \nabla+1: W_{D}^{1, p^{\prime}}(\Omega) \rightarrow W_{D}^{-1, p^{\prime}}(\Omega)
$$

is the adjoint to

$$
-\nabla \cdot \mu \nabla+1: W_{D}^{1, p}(\Omega) \rightarrow W_{D}^{-1, p}(\Omega)
$$

with respect to the sesquilinear pairing. Hence, 5.9 is a topological isomorphism iff (5.10) is. Thus, the interval $I$ must be of the form $I=] \frac{q}{q-1}, q[$ for some $q>2$.

(iii) It is well-known that the interval $I$ depends on the domain $\Omega$ (see [36, [8]) as well as on $\mu$ (see [53] or [52]), and on $D$ (see [55]). The most important point is that the length of $I$ may be arbitrarily small, see [10, Ch. 4] for a striking example. Even in smooth situations it cannot be expected that 4 belongs to $I$, as the pioneering counterexample in 58 ] shows.

(iv) If $\mathcal{M}$ is a set of coefficient functions $\mu$ with a common $L^{\infty}$ bound and a common ellipticity constant, then one can find a common open interval $I_{\mathcal{M}}$ around 2 , such that (5.7) is a topological isomorphism for all $\mu \in \mathcal{M}$ and all $p \in I_{\mathcal{M}}$. Finally, one has

$$
\sup _{p \in I_{\mathcal{M}}} \sup _{\mu \in \mathcal{M}}\left\|(-\nabla \cdot \mu \nabla+1)^{-1}\right\|_{\mathcal{L}\left(W_{D}^{-1, p} ; W_{D}^{1, p}\right)}<\infty .
$$

The proof of this is completely analogous to [27, Thm. 1].

(v) It is interesting to observe that in the case of two space dimensions Theorem $5.6 \mathrm{im}$ mediately implies the Hölder continuity of the solution as long as the right hand side belongs to a space $W_{D}^{-1, p}(\Omega)$ with $p>2$. The question arises whether this remains true in higher dimensions, for $p$ exceeding the corresponding space dimension - despite the fact that the gradient of the solution does only admit integrability a bit more than 2 in general. We will prove - by entirely different methods - in a forthcoming paper [11] that this is indeed the case.

Corollary 5.8. Let Assumptions 2.2. 3.1 and 5.4 be satisfied and let I denote the interval guaranteed by Theorem 5.6. Then the following holds

(i) The operator

$$
-\nabla \cdot \mu \nabla+\lambda: W_{D}^{1, p}(\Omega) \rightarrow W_{D}^{-1, p}(\Omega)
$$

is a topological isomorphism for all $p \in I \cap[2, \infty[$, if $-\lambda \in \mathbb{C}$ is not an eigenvalue of $-\nabla \cdot \mu \nabla$.

(ii) If 0 is the only constant function in the space $W_{D}^{1,2}(\Omega)$, then 0 is not an eigenvalue of $-\nabla \cdot \mu \nabla$.

Proof. (i) According to Remark 3.2 (iii) the embeddings $W_{D}^{1, p}(\Omega) \hookrightarrow L^{p}(\Omega) \hookrightarrow W_{D}^{-1, p}(\Omega)$ are compact. Thus (5.11) can only fail to be an isomorphism, if $-\lambda$ is an eigenvalue for $-\nabla \cdot \mu \nabla$, according to the Riesz-Schauder theory, cf. [41, Ch. III.6.8]. Observe that an eigenvalue for $-\nabla \cdot \mu \nabla$, when considered on $W_{D}^{-1, p}(\Omega)$ for $p>2$ is automatically an eigenvalue when $-\nabla \cdot \mu \nabla$ is considered on $W_{D}^{-1,2}(\Omega)$.

(ii) Assume that this is false, and let $w \in \operatorname{dom}_{W_{D}^{-1, p}(\Omega)}(\nabla \cdot \mu \nabla) \subset W_{D}^{1,2}(\Omega)$ be the corresponding eigenfunction. Then, testing the equation $-\nabla \cdot \mu \nabla w=0$ by $w$, one gets

$$
0=\langle-\nabla \cdot \mu \nabla w, w\rangle \geq c \int_{\Omega}\|\nabla w\|^{2} \mathrm{dx},
$$

thanks to the ellipticity of $\mu$. Hence, $w$ has to be constant on $\Omega$, and, consequently, must be 0 by supposition. 
Remark 5.9. $W_{D}^{1,2}(\Omega)$ does not contain any nonzero constant function if $D$ is a $(d-1)$-set and the boundary around only one point in $D$ possesses a bi-Lipschitzian chart around.

5.2. Analytic semigroups. In the sequel we are going to show how to exploit the elliptic regularity result for proving resolvent estimates for the operators $-\nabla \cdot \mu \nabla$, which assure the generator property for an analytic semigroup on suitable spaces $W_{D}^{-1, p}(\Omega)$. It is well known that this property allows to solve parabolic equations like

$$
u^{\prime}-\nabla \cdot \mu \nabla u=f ; \quad u(0)=u_{0},
$$

where the right hand side $f$ depends Hölder continuously on time (or even suitably on the solution $u$ itself), see [47] or [32. Since we proceed very similar to [27] we do not point out all details but refer to that paper.

Theorem 5.10. Let Assumptions 2.2. 4.10 and 5.4 be satisfied. Suppose, additionally, that $\bar{\Omega} \subset \mathbb{R}^{d}$ is a d-set. Then the following assertions hold true.

(i) There is an open interval $J$ containing 2 , such that the operator $\nabla \cdot \mu \nabla$ generates an analytic semigroup on $W_{D}^{-1, p}(\Omega)$, as long as $p \in J$.

(ii) If $\mathcal{M}$ is a set of coefficient functions $\mu$ with common $L^{\infty}$ bound and common ellipticity constant, one can find - in the spirit of [i( ) - a common interval $J_{\mathcal{M}}$ for all these $\mu \in \mathcal{M}$.

(iii) There is an open interval $J_{\mathcal{M}}$ containing 2 such that for all $p \in J_{\mathcal{M}}$ one has resolvent estimates like

$$
\left\|(-\nabla \cdot \mu \nabla+1+\lambda)^{-1}\right\|_{\mathcal{L}\left(W_{D}^{-1, p}(\Omega)\right)} \leq \frac{c}{1+|\lambda|},
$$

which are uniform in $\mu \in \mathcal{M}, p \in J_{\mathcal{M}}$ and $\lambda \in \mathbb{C}_{+}:=\{\vartheta \in \mathbb{C}: \operatorname{Re}(\vartheta) \geq 0\}$, i.e. the same constant $c$ may be taken for all these parameters.

Proof. Assertion (iii) implies points (i) and (ii)] so we concentrate on this. Concerning the $p$ 's above 2 one proceeds exactly as in [27]: Assumption 4.10 provides an extension operator $\mathfrak{E}$ which acts continuously between the spaces $W_{D}^{1, p}(\Omega)$ and $W_{D}^{1, p}\left(\mathbb{R}^{d}\right)$, cf. Theorem 4.14 . This leads, via Corollary 5.8 to a (nontrivial) interval $I_{0}:=\left[2, p_{0}\right]$, such that 5.11) is a topological isomorphism for all $p \in I_{0}$ and all $\lambda \in \mathbb{C}_{+}$. In a next step, to the operators $-\nabla \cdot \mu \nabla$ we will associate operators on the extended domain $\widetilde{\Omega}:=\Omega \times] 0,1[$. The 'extended' boundary part $\widetilde{\Gamma}$ we define as $\widetilde{\Gamma}:=\Gamma \times] 0,1[$, thus obtaining

$$
\widetilde{D}:=\partial \widetilde{\Omega} \backslash \widetilde{\Gamma}=(\bar{\Omega} \times\{0,1\}) \cup(D \times] 0,1[)=(\bar{\Omega} \times\{0,1\}) \cup(D \times[0,1]) .
$$

Since $\bar{\Omega}$ is a $d$-set and $D$ is a $(d-1)$-set by supposition, it is clear that $\widetilde{D}$ is a $d$-set. Moreover, it is not hard to see that $\widetilde{\Gamma}$ satisfies (mutatis mutandis) the condition in Assumption 4.10

The following considerations can be carried out in detail in exactly the same way as in [27, and we give here only a short summary of the main steps. As in [27, for every $\lambda \in \mathbb{C}_{+}$and $\mu \in \mathcal{M}$, one defines a coefficient function $\widetilde{\mu}$ on $\widetilde{\Omega}$ in the following manner: Let $\mu^{\bullet}$ be the $L^{\infty}$ bound for the coefficient function $\mu$ and $\mu_{\bullet}$ its ellipticity constant. Then we introduce the coefficient function for the auxiliary divergence operator on $\widetilde{\Omega}$ by

$$
\tilde{\mu}_{j, k}(\mathrm{x}, t)= \begin{cases}\left(1-\frac{\mu_{\bullet}}{2 \mu^{\bullet}} \operatorname{sign}(\operatorname{Im}(\lambda)) i\right) \mu_{j, k}(\mathrm{x}), & \text { if } j, k \in\{1, \ldots, d\}, \\ 0 & \text { if } j=d+1 \text { or } k=d+1, \\ \frac{\lambda}{|\lambda|}\left(\mu^{\bullet}-\frac{\mu_{\bullet}}{2} \operatorname{sign}(\operatorname{Im}(\lambda)) i\right), & \text { if } j=k=d+1 .\end{cases}
$$

One easily observes that all these coefficient functions admit $L^{\infty}$ bounds and ellipticity constants that are uniform in $\lambda$. Thus, Remark 5.7 (iv)] applies to the operators $-\nabla \cdot \widetilde{\mu} \nabla+1$. This gives an interval $I_{1}:=\left[2, p_{1}\right]$ such that the norms of the operators $(-\nabla \cdot \widetilde{\mu} \nabla+1)^{-1}: W_{\widetilde{D}}^{-1, p}(\widetilde{\Omega}) \rightarrow$ $W_{\widetilde{D}}^{1, p}(\widetilde{\Omega})$ are bounded, uniformly in $\lambda \in \mathbb{C}_{+}$and in $p \in I_{1}$. 
One associates to the problem $(-\nabla \cdot \mu \nabla+1+\lambda) u=f$ a problem $(-\nabla \cdot \widetilde{\mu} \nabla+1) u_{\lambda}=f_{\lambda}$ and exploits the (uniform) regularity properties of the operators $-\nabla \cdot \widetilde{\mu} \nabla+1$ for an estimate

$$
\|u\|_{W_{D}^{1, p}(\Omega)} \leq c\|f\|_{W_{D}^{-1, p}(\Omega)},
$$

where $c$ is independent from $f$ and $\lambda \in \mathbb{C}_{+}$. We already know the isomorphism property

$$
-\nabla \cdot \mu \nabla+1+\lambda: W_{D}^{1, p}(\Omega) \rightarrow W_{D}^{-1, p}(\Omega),
$$

thus 5.15 may be expressed as

$$
\sup _{\lambda \in \mathbb{C}_{+}}\left\|(-\nabla \cdot \mu \nabla+1+\lambda)^{-1}\right\|_{\mathcal{L}\left(W_{D}^{-1, p}(\Omega) ; W_{D}^{1, p}(\Omega)\right)}<\infty
$$

for all $p \in I_{0} \cap I_{1}$.

Finally, (5.16) allows us to deduce the estimate

$$
\begin{aligned}
& \sup _{\lambda \in \mathbb{C}_{+}}|\lambda|\left\|(-\nabla \cdot \mu \nabla+1+\lambda)^{-1}\right\|_{\mathcal{L}\left(W_{D}^{-1, p}(\Omega)\right)} \\
= & \sup _{\lambda \in \mathbb{C}_{+}}\left\|\lambda(-\nabla \cdot \mu \nabla+1+\lambda)^{-1}\right\|_{\mathcal{L}\left(W_{D}^{-1, p}(\Omega)\right)} \\
= & \sup _{\lambda \in \mathbb{C}_{+}}\left\|1-(-\nabla \cdot \mu \nabla+1)(-\nabla \cdot \mu \nabla+1+\lambda)^{-1}\right\|_{\mathcal{L}\left(W_{D}^{-1, p}(\Omega)\right)} \\
\leq & 1+\|-\nabla \cdot \mu \nabla+1\|_{\mathcal{L}\left(W_{D}^{1, p}(\Omega) ; W_{D}^{-1, p}(\Omega)\right)} \sup _{\lambda \in \mathbb{C}_{+}}\left\|(-\nabla \cdot \mu \nabla+1+\lambda)^{-1}\right\|_{\mathcal{L}\left(W_{D}^{-1, p}(\Omega) ; W_{D}^{1, p}(\Omega)\right)} \\
< & \infty
\end{aligned}
$$

for all $p \in I_{0} \cap I_{1}$.

The case $p<2$ can be treated as follows: first, one gets the following resolvent estimate on $W_{D}^{1, p}(\Omega)$ for $p>2$ :

$$
\begin{aligned}
& \left\|(-\nabla \cdot \mu \nabla+1+\lambda)^{-1}\right\|_{\mathcal{L}\left(W_{D}^{1, p}(\Omega)\right)} \\
= & \left\|(-\nabla \cdot \mu \nabla+1)^{-1}(-\nabla \cdot \mu \nabla+1+\lambda)^{-1}(-\nabla \cdot \mu \nabla+1)\right\|_{\mathcal{L}\left(W_{D}^{1, p}(\Omega)\right)} \\
\leq & \left\|(-\nabla \cdot \mu \nabla+1)^{-1}\right\|_{\mathcal{L}\left(W_{D}^{-1, p}(\Omega) ; W_{D}^{1, p}(\Omega)\right)} \\
& \left\|(-\nabla \cdot \mu \nabla+1+\lambda)^{-1}\right\|_{\mathcal{L}\left(W_{D}^{-1, p}(\Omega)\right)} \\
\times & \|-\nabla \cdot \mu \nabla+1\|_{\mathcal{L}\left(W_{D}^{1, p}(\Omega) ; W_{D}^{-1, p}(\Omega)\right)}
\end{aligned}
$$

Since the first and third factor are finite, one can use (5.12). Then, considering the adjoint of $(-\nabla \cdot \mu \nabla+1+\lambda)^{-1}$, which is nothing else but $\left(-\nabla \cdot \mu^{*} \nabla+1+\bar{\lambda}\right)^{-1}$ on $W_{D}^{-1, p^{\prime}}(\Omega)$, one obtains the assertion for $p<2$.

Remark 5.11. One could take the suppositions in Theorem 5.10 even more general. What in fact is needed is that also the spaces $W_{\widetilde{D}}^{1, p}(\widetilde{\Omega})$ possess extension operators. This follows in case of Assumption 4.10 in a peculiarly simple way since it is self-reproducing when passing to the set $\Omega \times] 0,1[$.

\section{Elliptic REgularity FOR Systems}

In this section we apply the interpolation property of the $W^{1, p}$-spaces in order to derive $p$ estimates for linear elliptic operators acting on vector-valued functions. Here, for each component a different Dirichlet boundary might be prescribed. To be more precise, we assume the following

(A1) $\Omega \subset \mathbb{R}^{d}$ is a bounded domain and for $1 \leq i \leq m$ the sets $D_{i} \subset \partial \Omega$ are closed $(d-1)$-sets. Let $D:=\bigcap_{i=1}^{m} D_{i}$ and $\Gamma:=\partial \Omega \backslash D$. It is assumed that $\Omega$ and $\Gamma$ satisfy Assumption 3.1. cf. Subsection 4.2. 
For $p \in[1, \infty)$ we introduce the space

$$
\mathbb{W}_{D}^{1, p}(\Omega)=\prod_{i=1}^{m} W_{D_{i}}^{1, p}(\Omega)
$$

and its dual $\mathbb{W}_{D}^{-1, p^{\prime}}(\Omega)$ for $\frac{1}{p}+\frac{1}{p^{\prime}}=1$, Furthermore, we define the operator $\mathcal{L}_{p}: \mathbb{W}_{D}^{1, p}(\Omega) \rightarrow$ $L^{p}\left(\Omega ; \mathbb{C}^{m} \times \mathbb{C}^{m \times d}\right)$ by $\mathcal{L}_{p}(u)=(u, \nabla u)$. Given a complex valued coefficient function $\mathbb{A} \in$ $L^{\infty}\left(\Omega ; \operatorname{Lin}\left(\mathbb{C}^{m} \times \mathbb{C}^{m \times d}, \mathbb{C}^{m} \times \mathbb{C}^{m \times d}\right)\right)$, we investigate differential operators of the type

$$
\mathcal{A}: \mathbb{W}_{D}^{1, p}(\Omega) \rightarrow \mathbb{W}_{D}^{-1, p}(\Omega), \quad \mathcal{A}=\mathcal{L}_{p^{\prime}}^{*} \mathbb{A} \mathcal{L}_{p}
$$

The corresponding weak formulation on $\mathbb{W}_{D}^{1,2}(\Omega) \operatorname{reads}\langle\mathcal{A}(u), v\rangle=\int_{\Omega} \mathbb{A}(\stackrel{u}{\nabla u}): \overline{(\stackrel{v}{\nabla v})} d \mathrm{x}$ for $u, v \in \mathbb{W}_{D}^{1,2}(\Omega)$, where

$$
\left(b_{1}, B_{1}\right):\left(b_{2}, B_{2}\right)=\sum_{i=1}^{m} b_{1}^{i} b_{2}^{i}+\sum_{j=1}^{m} \sum_{k=1}^{d} B_{1}^{j k} B_{2}^{j k}
$$

for $\left(b_{1}, B_{1}\right),\left(b_{2}, B_{2}\right) \in \mathbb{C}^{m} \times \mathbb{C}^{m \times d}$. It is assumed that the operator $\mathcal{A}$ is elliptic. More precisely, we assume that

(A2) There is a constant $\kappa>0$, such that for all $v \in \mathbb{W}_{D}^{1,2}(\Omega)$ it holds $\operatorname{Re}\langle\mathcal{A} v, v\rangle \geq \kappa\|v\|_{\mathbb{W}^{1,2}(\Omega)}^{2}$.

Remark 6.1. We recall that in the case of systems of partial differential equations the positivity property formulated in (A2) in general does not imply that the coefficient tensor belonging to the principle part of the differential operator is positive definite. In general, this coefficient tensor only satisfies the weaker Legendre-Hadamard condition, cf. [19]: Assume that (A2) is satisfied for $\mathbb{A}=\left(\begin{array}{l}\mathbb{A}_{11} \mathbb{A}_{12} \\ \mathbb{A}_{21}\end{array} \mathbb{A}_{22}\right)$, where $\mathbb{A}_{22} \in \operatorname{Lin}\left(\mathbb{C}^{m \times d}, \mathbb{C}^{m \times d}\right)$ corresponds to the principal part of the operator $\mathcal{A}$. Then there exists a constant $c_{\kappa}>0$, such that for all $\xi \in \mathbb{C}^{m}$ and $\eta \in \mathbb{C}^{d}$ it holds

$$
\operatorname{Re}\left(\mathbb{A}_{22} \xi \otimes \eta: \overline{\xi \otimes \eta}\right) \geq c_{\kappa}|\xi|^{2}|\eta|^{2} .
$$

Theorem 6.2. Let (A1) and (A2) be satisfied. Then there exists an open interval $J$ containing 2 , such that for all $q \in J$ the operator $\mathcal{A}: \mathbb{W}_{D}^{1, q}(\Omega) \rightarrow \mathbb{W}_{D}^{-1, q}(\Omega)$ is a topological isomorphism.

Proof. Exactly the same arguments as in the proof of Theorem 5.6 can be applied.

If in addition the operator $\mathcal{A}$ satisfies a certain symmetry relation, then the interval $J$ can be determined uniformly for classes of coefficient tensors satisfying uniform upper bounds and ellipticity properties.

(A3) For all $u, v \in \mathbb{W}_{D}^{1,2}(\Omega)$ it holds $\langle\mathcal{A} u, v\rangle=\overline{\langle\mathcal{A} v, u\rangle}$.

Theorem 6.3. Let (A1) be satisfied and let $\mathcal{M}$ be a set of coefficient tensors fulfilling (A2) and (A3) with a uniform upper $L^{\infty}$-bound and a common lower bound for the ellipticity constant $\kappa$ in (A2). Then, there exists an open interval $J_{\mathcal{M}}$ containing 2 , such that for all $p \in J_{\mathcal{M}}$ and all $\mathbb{A} \in \mathcal{M}$ the corresponding operator $\mathcal{A}$ is a topological isomorphism between $\mathbb{W}_{D}^{1, p}(\Omega)$ and $\mathbb{W}_{D}^{-1, p}(\Omega)$. Moreover, there exists a constant $c_{\mathcal{M}}>0$ such that for all $f \in \mathbb{W}_{D}^{-1, p}(\Omega)$ we have

$$
\sup \left\{\left\|\mathcal{A}^{-1}(f)\right\|_{\mathbb{W}_{1}^{1, p}(\Omega)} ; p \in J_{\mathcal{M}}, \mathbb{A} \in \mathcal{M}\right\} \leq c_{\mathcal{M}}\|f\|_{\mathbb{W}_{D}^{-1, p}(\Omega)} .
$$

Remark 6.4. In the case of scalar equations, i.e. $m=1$, the previous theorem is also valid for operators $\mathcal{A}$ which do not satisfy (A3), (see Remark 5.7 (iv)). Similar arguments as in the scalar case can be applied to the vectorial case without assuming (A3) provided that the coefficient tensor $\mathbb{A}_{22}$ satisfies $(6.1)$ for all $B \in \mathbb{C}^{m \times d}$ and not only for $B=\xi \otimes \eta$. In this case, the proof of the uniform bound (6.2) relies on certain estimates that are derived using the positivity of the coefficient-tensors (see [27]). In the general non-symmetric vector valued case, we do not see how the proof can be generalised, if only the weaker positivity (6.1) is assumed. In the case studied in 
Theorem 6.3 we derive estimates for the corresponding operators directly (and not pointwise for the coefficients) and use the fact that for self-adjoint operators on a Hilbert space $\mathbb{H}$ the operator norm is given by $\|T\|_{\mathrm{op}}=\sup \{|\langle T a, a\rangle| ; a \in \mathbb{H},\|a\| \leq 1\}$.

Proof. Let $\mathcal{P}: \mathbb{W}_{D}^{1,2}(\Omega) \rightarrow \mathbb{W}_{D}^{-1,2}(\Omega)$ be defined by $\mathcal{P}=\mathcal{L}^{*} \mathcal{L}$. Due to Theorem 6.2 there exist $q_{0}^{*}<2<q_{1}^{*}$ such that for all $p \in\left[q_{0}^{*}, q_{1}^{*}\right]$ the operator $\mathcal{P}$ is a topological isomorphism between $\mathbb{W}_{D}^{1, p}(\Omega)$ and $\mathbb{W}_{D}^{-1, p}(\Omega)$. This implies that for all $t>0$ and $p \in\left[q_{0}^{*}, q_{1}^{*}\right]$ the operator $\mathcal{Q}_{t}$, given by $\mathcal{Q}_{t}=\mathcal{P}^{-1}(\mathcal{P}-t \mathcal{A})$ is a bounded linear operator from $\mathbb{W}_{D}^{1, p}(\Omega)$ to $\mathbb{W}_{D}^{1, p}(\Omega)$. In a first step, we will show that there exist $t_{0}>0$ and $q_{0}, q_{1} \in\left[q_{0}^{*}, q_{1}^{*}\right]$ with $q_{0}<2<q_{1}$, such that

$$
\sup _{p \in\left[q_{0}, q_{1}\right]}\left\|\mathcal{Q}_{t_{0}}\right\|_{\mathrm{op}, p} \leq \iota<1
$$

where $\left\|\mathcal{Q}_{t_{0}}\right\|_{\mathrm{op}, p}$ denotes the operator norm with respect to the space $\mathbb{W}_{D}^{1, p}(\Omega)$. In the second step, the uniform estimate 6.2 is derived from 6.3.

We start the investigation with $p=2$. Observe that the standard inner product on $\mathbb{W}_{D}^{1,2}(\Omega)$ satisfies $(u, v)_{1,2}=(\mathcal{L} u, \mathcal{L} v)_{0,2}=\langle\mathcal{P}(u), v\rangle$. Hence, by (A3) the following identities are valid for $u, v \in \mathbb{W}_{D}^{1,2}(\Omega):$

$$
\left(\mathcal{Q}_{t} u, v\right)_{1,2}=\langle(\mathcal{P}-t \mathcal{A}) u, v\rangle=\overline{\langle(\mathcal{P}-t \mathcal{A}) v, u\rangle}=\overline{\left(\mathcal{P}^{-1}(\mathcal{P}-t \mathcal{A}) v, u\right)_{1,2}}=\left(u, \mathcal{Q}_{t} v\right)_{1,2}
$$

This shows that $\mathcal{Q}_{t}$ is self adjoint on $\mathbb{W}_{D}^{1,2}(\Omega)$. Moreover, taking into account the upper bound $M$ of the coefficient matrix $\mathbb{A}$ and the uniform ellipticity property, the following estimates are valid for all $u \in \mathbb{W}_{D}^{1,2}(\Omega)$ :

$$
\begin{aligned}
& \left(\mathcal{Q}_{t} u, u\right)_{1,2}=\langle(\mathcal{P}-t \mathcal{A}) u, u\rangle \geq(1-t M)\|u\|_{\mathbb{W}^{1,2}(\Omega)}^{2} \\
& \left(\mathcal{Q}_{t} u, u\right)_{1,2} \leq(1-t \kappa)\|u\|_{\mathbb{W}^{1,2}(\Omega)}^{2} .
\end{aligned}
$$

Thus, the operator norm $\left\|\mathcal{Q}_{t}\right\|_{\mathrm{op}, 2}$ with respect to $\mathbb{W}_{D}^{1,2}(\Omega)$ can be estimated as

$$
\begin{aligned}
\left\|\mathcal{Q}_{t}\right\|_{\mathrm{op}, 2} & =\sup \left\{\left|\left\langle\mathcal{Q}_{t} u, u\right\rangle\right| ; u \in \mathbb{W}_{D}^{1,2}(\Omega),\|u\|_{\mathbb{W}^{1,2}(\Omega)} \leq 1\right\} \\
& \leq \max \{|1-t M|,|1-t \kappa|\} .
\end{aligned}
$$

Hence, the operator $\mathcal{Q}_{t}$ is a strict contraction provided that $\left.t \in\right] 0,2 / M\left[\right.$. We choose now $t_{0}=$ $2 /(\kappa+M)$ and define $\widetilde{\iota}=1-t_{0} \kappa=(M-\kappa) /(M+\kappa)$. With this, we have $\left\|\mathcal{Q}_{t_{0}}\right\|_{\mathrm{op}, 2} \leq \widetilde{\imath}<1$.

For $p \in\left[2, q_{1}^{*}\right]$, interpolation theory gives the estimate $\left\|\mathcal{Q}_{t_{0}}\right\|_{\mathrm{op}, p} \leq \widetilde{\iota}^{1-\theta}\left\|\mathcal{Q}_{t_{0}}\right\|_{\mathrm{op}, q_{1}^{*}}^{\theta}$, where $1 / p=(1-\theta) / 2+\theta / q_{1}^{*}$. Hence, there exist $\left.\iota_{1} \in\right] 0,1\left[\right.$ and $\left.\left.q_{1} \in\right] 2, q_{1}^{*}\right]$ such that for all $p \in\left[2, q_{1}\right]$ it holds $\left\|\mathcal{Q}_{t_{0}}\right\|_{\mathrm{op}, p} \leq \iota_{1}$. Similar arguments applied to the interval $\left[q_{0}^{*}, 2\right]$ finally imply (6.3).

Now, we proceed analogously to the arguments in the proof of Theorem 1 in [26]: Since the operator $\mathcal{Q}_{t_{0}}$ is a contraction on $\mathbb{W}_{D}^{1, p}(\Omega)$, for every $f \in \mathbb{W}_{D}^{-1, p}(\Omega)$ the operator $v \mapsto \mathcal{Q}_{t_{0}}(v)+$ $t_{0} \mathcal{P}^{-1} f$ has a unique fixed point $u_{f}$. Observe that $u_{f}$ satisfies $\mathcal{A} u_{f}=f$. Hence, for all $p \in\left[p_{0}, p_{1}\right]$ the operator $\mathcal{A}$ is a topological isomorphism with respect to $\mathbb{W}_{D}^{1, p}(\Omega)$. Finally, since

$$
\left\|u_{f}\right\|_{\mathbb{W}^{1, p}(\Omega)}=\left\|\mathcal{Q}_{t_{0}} u_{f}+t_{0} \mathcal{P}^{-1} f\right\|_{\mathbb{W}^{1, p}(\Omega)} \leq \iota\left\|u_{f}\right\|_{\mathbb{W}^{1, p}(\Omega)}+t_{0} c_{q_{0}^{*}, q_{1}^{*}}\|f\|_{\mathbb{W}_{D}^{-1, p}(\Omega)},
$$

the operator norm of $\mathcal{A}^{-1}$ is uniformly bounded on $\left[q_{0}, q_{1}\right]$, which is 6.2.

Example 6.5. The equations of linear elasticity as well as the Cosserat-model fit into this framework. In the case of linear elasticity, the vector-function $u: \Omega \rightarrow \mathbb{R}^{d}$ (i.e. $m=d$ ) denotes the displacement field. Typically, the Dirichlet-boundary is the same for all components of $u$. Hence, we define $\mathbb{W}_{D}^{1, p}(\Omega)=\prod_{i=1}^{d} W_{D}^{1, p}(\Omega)$, where $D \subset \partial \Omega$ is a closed $(d-1)$-set. The operator of linear elasticity is defined through the form $\langle\mathcal{A} u, v\rangle=\int_{\Omega} \mathbf{C} e(u): e(v) \mathrm{dx}$ for $u, v \in \mathbb{W}_{D}^{1,2}(\Omega)$, Here, $e(u)=\frac{1}{2}\left(\nabla u+\nabla u^{\top}\right)$ is the symmetrised gradient and $\mathbf{C} \in L^{\infty}\left(\Omega ; \operatorname{Lin}\left(\mathbb{R}_{\mathrm{sym}}^{d \times d}, \mathbb{R}_{\mathrm{sym}}^{d \times d}\right)\right)$ denotes 
the fourth order elasticity tensor. It is assumed that $\mathbf{C}$ is symmetric and positive definite on the symmetric matrices: for all $F_{1}, F_{2} \in \mathbb{R}_{\text {sym }}^{d \times d}$ it holds

$$
\mathbf{C} F_{1}: F_{2}=\mathbf{C} F_{2}: F_{1}, \quad \mathbf{C} F_{1}: F_{1} \geq c_{\kappa}\left|F_{1}\right|^{2} \text {. }
$$

In order to have Korn's second inequality at our disposal, in addition to (A1) we assume that $\Omega$ is a Lipschitz domain. Korn's second inequality states that the standard norm in $\mathbb{W}_{D}^{1,2}(\Omega)$ and the norm $|\|u\||:=\|u\|_{L^{2}(\Omega)}+\|e(u)\|_{L^{2}(\Omega)}$ are equivalent, cf. [18] and the references therein. Moreover, if $\mathcal{H}_{d-1}(D)>0$, then standard arguments relying on the compact embedding of $\mathbb{W}_{D}^{1,2}(\Omega)$ in $L^{2}(\Omega)$ show that also Korn's first inequality is valid and assumption (A2) is satisfied. Hence, Theorems 6.2 and 6.3 are applicable.

In the Cosserat models, additionally to the displacement fields the skew symmetric Cosseratmicrorotation-tensor $R \in \mathbb{R}_{\text {skew }}^{3 \times 3}$ plays a role. Via the relation

$$
\operatorname{axl} R:=\operatorname{axl}\left(\begin{array}{ccc}
0 & r_{1} & r_{2} \\
-r_{1} & 0 & r_{3} \\
-r 2 & -r 3 & 0
\end{array}\right):=\left(\begin{array}{c}
-r_{3} \\
r_{2} \\
-r_{1}
\end{array}\right)
$$

$\mathbb{R}_{\text {skew }}^{3 \times 3}$ is identified with $\mathbb{R}^{3}$. Assume that $D_{\mathrm{el}}, D_{\mathrm{R}} \subset \partial \Omega$ are nonempty, closed 2-sets describing the Dirichlet boundary for the displacements and the tensor $R$, respectively. The state space is defined as $\mathbb{W}_{D}^{1, p}(\Omega)=\prod_{i=1}^{3} W_{D_{\mathrm{el}}}^{1, p}(\Omega) \times \prod_{i=1}^{3} W_{D_{\mathrm{R}}}^{1, p}(\Omega)$. A typical differential operator occurring in the theory of Cosserat models is given by the following weak form for $(u, R),(v, Q) \in \mathbb{W}_{D}^{1,2}(\Omega)$ :

$$
\begin{aligned}
\left\langle\mathcal{A}\left(\begin{array}{c}
u \\
R
\end{array}\right),\left(\begin{array}{l}
v \\
Q
\end{array}\right)\right\rangle=\int_{\Omega} 2 \mu e(u): e(v) & +\lambda \operatorname{div} u \operatorname{div} v \\
& +2 \mu_{c} \operatorname{skew}(\nabla u-R): \operatorname{skew}(\nabla v-Q)+\gamma \nabla \operatorname{axl} R: \nabla \operatorname{axl} Q \mathrm{~d} x .
\end{aligned}
$$

If in addition to (A1) the domain is a Lipschitz domain and if for the Lamé-constants $\lambda, \mu$, the Cosserat-couple modulus $\mu_{c}$ and the parameter $\gamma$ it holds $\mu>0,2 \mu+3 \lambda>0, \mu_{c} \geq 0$ and $\gamma>0$, then condition (A2) is satisfied, see [56, 42], where also more general situations are discussed. Obviously, (A3) is satisfied as well, and hence Theorems 6.2 and 6.3 are applicable.

Remark 6.6. We finally remark that on the basis of the previous example the results from 33 for nonlinear elasticity models can be extended to the situation discussed here by repeating the arguments in [33, Section 3].

\section{Applications}

In this chapter we intend to indicate possible applications, which were the original motivation for this work.

It is more or less clear that the results of this paper cry for applications primarily in spatially two-dimensional elliptic/parabolic problems. We suggest that in almost all applications resting on 26] the geometric conditions can be relaxed to those of this paper, and the results still hold, (see e.g. [46, [9], 39], 54, 6], 15], 21], [16], 40], 35] to name only a few).

Moreover, the generator property for an analytic semigroup gives the opportunity to deal also with parabolic problems. When employing the main result from [13 and then applying the classical semilinear theory, see e.g. [32, Ch. 3], one should be able to treat also semilinear ones. Generally, the $W_{D}^{-1, q}$-calculus allows for right hand sides of the equations which contain distributional objects as e.g. surface densities which still belong to the space $W_{D}^{-1, q}(\Omega)$. In particular, in the $2 d$-case one may even admit functions in time which take their values in the space of Borel measures, since the space of these measures then continuously embeds into any space $W_{D}^{-1, q}(\Omega)$ with $q<2$, compare also [2].

Moreover, the elliptic regularity result enables a simpler treatment of problems which include quadratic gradient terms: the a priori knowledge $\nabla u \in L^{q}$ with $q>2$ improves the standard 
information $|\nabla u|^{2} \in L^{1}$ to $|\nabla u|^{2} \in L^{r}$ with $r>1$ - what makes the analysis of such problems easier, compare [35, 43].

At the end, let us sketch an idea how one can exploit the gain in elliptic regularity in a rather unexpected direction: Let $q>2$ be a number such that (5.7) is a topological isomorphism and (5.7) is also a topological isomorphism if $\mu$ is there replaced by the adjoint coefficient function, then providing the adjoint operator in $L^{2}(\Omega)$. We abbreviate $A:=\left.\nabla \cdot \mu \nabla\right|_{L^{2}(\Omega)}$. As in [13] one can prove that the semigroup operators $e^{t A}$ possess kernels which admit upper Gaussian estimates. Obviously, these kernels are bounded, and, consequently, all semigroup operators are Hilbert-Schmidt and even nuclear. Consequently, $e^{\frac{t}{3} A}: L^{2}(\Omega) \rightarrow L^{2}(\Omega)$ admits a representation

$$
e^{\frac{t}{3} A} \psi=\sum_{j} \lambda_{j}\left\langle\psi, f_{j}\right\rangle_{L^{2}(\Omega)} g_{j}
$$

with $\left\|f_{j}\right\|_{L^{2}(\Omega)}=\left\|g_{j}\right\|_{L^{2}(\Omega)}=1$ and $\sum_{j}\left|\lambda_{j}\right|<\infty$, see [44, Thm. 1.b.3]. Hence, $e^{t A}$ admits the following representation via an integral kernel.

$$
e^{t A}=\sum_{j} \lambda_{j}\left\langle e^{\frac{t}{3} A} \cdot, f_{j}\right\rangle_{L^{2}(\Omega)} e^{\frac{t}{3} A} g_{j}=\sum_{j} \lambda_{j} e^{\frac{t}{3} A} g_{j} \otimes \overline{e^{\frac{t}{3} A^{\star}} f_{j}} .
$$

Let us estimate the $W^{1, q}$-norm of the elements $e^{\frac{t}{3} A} g_{j}$ and $e^{\frac{t}{3} A^{\star}} f_{j}$, respectively:

$$
\left\|e^{\frac{t}{3} A} g_{j}\right\|_{W_{D}^{1, q}(\Omega)} \leq\left\|(-A+1)^{-1}\right\|_{\mathcal{L}\left(L^{q}(\Omega) ; W_{D}^{1, q}(\Omega)\right)}\left\|e^{\frac{t}{6} A}\right\|_{\mathcal{L}\left(L^{2}(\Omega) ; L^{q}(\Omega)\right)}\left\|(-A+1) e^{\frac{t}{6} A}\right\|_{\mathcal{L}\left(L^{2}(\Omega)\right)},
$$

since $\left\|g_{j}\right\|_{L^{2}(\Omega)}=1$. Let us discuss the factors on the right hand side: the first is finite due to our supposition on $q$ and the embedding $L^{q}(\Omega) \hookrightarrow W_{D}^{-1, q}(\Omega)$. The second is finite because the semigroup operators are integral operators with bounded kernels. The third factor is bounded because $A$ generates an analytic semigroup on $L^{2}(\Omega)$.

The estimate for $e^{\frac{t}{3} A^{\star}} f_{j}$ is quite analogous, this time investing the continuity of $\left(-A^{*}+1\right)^{-1}$ : $L^{q}(\Omega) \rightarrow W_{D}^{1, q}(\Omega)$. Bringing now into play the summability of the series $\sum_{j}\left|\lambda_{j}\right|$, one obtains the convergence of the series $\sum_{j} \lambda_{j} e^{\frac{t}{3} A} g_{j} \otimes \overline{e^{\frac{t}{3} A^{\star}} f_{j}}$ in the space $W^{1, q}(\Omega \times \Omega)$. Thus, the semigroup operators have kernels which are even from $W^{1, q}(\Omega \times \Omega)$ with $q>2$. We will discuss the consequences of this in a forthcoming paper.

\section{Concluding Remarks}

Remark 8.1. (i) As the examples in Figure 1 and 2 suggest, admissible constellations for the domain $\Omega$ are finite unions of (suitable) Lipschitz domains, whose closures have nonempty intersections. Thus, generically, the boundary is the finite union of $(d-1)$ dimensional Lipschitz manifolds with the corresponding boundary measures.

(ii) The $W^{1, p}$-regularity result is also of use for the analysis of four-dimensional elliptic equations with right hand side from $W_{D}^{-1, p}(\Omega), p>4$. Namely, the information that the solution a priori belongs to a space $W_{D}^{1, q}$ with $q>2$, allows to localise the elliptic problem within the same class of right hand sides, cf. [28.

Acknowledgment In an ealier version of this paper we used Assumption 4.10 in order to establish our geometric setting in view of the extension operator, cf. [13. However, we learnt from a referee report on this paper that it should be possible to extend this to more general settings.

Secondly, we wish to thank Moritz Egert (Darmstadt) for pointing out to us Lemma 4.9 .

\section{REFERENCES}

[1] H. Amann: Nonhomogeneous linear and quasilinear elliptic and parabolic boundary value problems. In: H.J. Schmeisser et al. (eds.): Function spaces, differential operators and nonlinear analysis, vol. 133 of TeubnerTexte Math., pp. 9-126, Teubner, Stuttgart, 1993. 
[2] H. Amann: Linear parabolic problems involving measures. RACSAM Rev. R. Acad. Cienc. Exactas Fis. Nat. Ser. A Mat. 95 (2001), no. 1, 85-119.

[3] P. Auscher: On necessary and sufficient conditions for $L^{p}$-estimates of Riesz Transforms Associated to Elliptic Operators on $\mathbb{R}^{n}$ and related estimates. Mem. Amer. Math. Soc. 186 (2007), no. 871.

[4] K. Brewster, D. Mitrea, I. Mitrea, M. Mitrea: Extending Sobolev functions with partially vanishing traces from locally $(\varepsilon, \delta)$-domains and applications to mixed boundary value problems. arXiv No. 1208.4177.

[5] P.G. Ciarlet: The finite element method for elliptic problems. Vol 4 of Studies in Mathematics and its Applications, North Holland, Amsterdam-New York-Oxford, 1978.

[6] L. Consiglieri, M.C. Muniz: Existence of solutions for a free boundary problem in the thermoelectrical modelling of an aluminium electrolytic cell. European J. Appl. Math. 14 (2003), no 2, 201-216.

[7] D. Daners: Inverse positivity of general Robin problems on Lipschitz domains. Arch. Math. (Basel) 92 (2009), no. $1,57-69$.

[8] M. Dauge: Neumann and mixed problems on curvilinear polyhedra. Integral Equations Oper. Theory 15 (1992), no. 2, 227-261.

[9] P. Degond, S. Genieys, A. Jüngel: A steady-state system in non-equilibrium thermodynamics including thermal and electrical effects. Math. Methods Appl. Sci. 21 (1998), no. 15, 1399-1413.

[10] J. Elschner, J. Rehberg, G. Schmidt: Optimal regularity for elliptic transmission problems including $C^{1}$ interfaces. Interfaces Free Bound. 9 (2007) 233-252.

[11] A.F.M. ter Elst, R. Haller-Dintelmann, J. Rehberg: Hölder continuity for solutions to mixed boundary value problems. In preparation.

[12] A.F.M. ter Elst, M. Meyries, J. Rehberg: Parabolic equations with dynamical boundary conditions and source terms on interfaces. WIAS-Preprint No. 1711, arXiv No. 1206.0600. to appear in Annali Matematica Pura Applicata.

[13] A.F.M. ter Elst, J. Rehberg: $L^{\infty}$-estimates for divergence operators on bad domains. Anal. Appl. (Singap.) 10 (2012) 207-214.

[14] L.C. Evans, R.F. Gariepy: Measure theory and fine properties of functions. Studies in advanced mathematics, CRC Press, Boca Raton-New York-London-Tokyo, 1992.

[15] P. Fabrie, T. Gallouët: Modelling wells in porous media flow. Math. Models Methods Appl. Sci. 10 (2000), no. 5, 673-709.

[16] H. Gajewski, K. Gröger: Initial boundary value problems modelling heterogeneous semiconductor devices. In: B.W. Schulze, H. Triebel (eds.): Surveys on Analysis, Geometry and Mathematical Physics, vol. 117 of Teubner-Texte Math., pp. 4-53, Teubner, Leipzig, 1990.

[17] H. Gajewski, K. Gröger, K. Zacharias: Nichtlineare Operatorgleichungen und Operatordifferentialgleichungen. Akademie-Verlag, Berlin, 1974.

[18] G. Geymonat, P. Suquet: Functional spaces for Norton-Hoff materials. Math. Methods Appl. Sci. 8 (1986) $206-222$.

[19] M. Giaquinta, S. Hildebrandt: Calculus of Variations I. Springer Verlag, Berlin-Heidelberg, 1996.

[20] D. Gilbarg, N.S. Trudinger: Elliptic partial differential equations of second order, 2nd. Ed., Springer-Verlag, Berlin, 1983.

[21] A. Glitzky, R. Hünlich: Global estimates and asymptotics for electro-reaction-diffusion equations. Appl. Anal. 66 (1997) 205-226.

[22] J.A. Griepentrog: Linear elliptic boundary value problems with non-smooth data: Campanato spaces of functionals. Math. Nachr. 243 (2002) 19-42.

[23] J.A. Griepentrog: Maximal regularity for nonsmooth parabolic problems in Sobolev-Morrey spaces. Adv. Differential Equations 12 (2007), no. 9, 1031-1078.

[24] J.A. Griepentrog, K. Gröger, H.-C. Kaiser, J. Rehberg: Interpolation for function spaces related to mixed boundary value problems. Math. Nachr. 241 (2002) 110-120.

[25] J.A. Griepentrog, L. Recke: Linear elliptic boundary value problems with non-smooth data: normal solvability on Sobolev-Campanato spaces. Math. Nachr. 225 (2001) 39-74.

[26] K. Gröger: A $W^{1, p}$-estimate for solutions to mixed boundary value problems for second order elliptic differential equations. Math. Ann. 283 (1989) 679-687.

[27] K. Gröger, J. Rehberg: Resolvent estimates in $W^{-1, p}$ for second order elliptic differential operators in case of mixed boundary conditions. Math. Ann. 285 (1989), no. 1, 105-113.

[28] R. Haller-Dintelmann, C. Meyer, J. Rehberg, A. Schiela: Hölder continuity and optimal control for nonsmooth elliptic problems. Appl. Math. Optim. 60 (2009), no. 3, 397-428.

[29] R. Haller-Dintelmann, J. Rehberg: Maximal parabolic regularity for divergence operators including mixed boundary conditions. J. Differential Equations 247 (2009), no. 5, 1354-1396.

[30] R. Haller-Dintelmann, J. Rehberg: Coercivity for elliptic operators and positivity of solutions on Lipschitz domains. Arch. Math. (Basel) 95 (2010), no. 5, 457-468. 
[31] R. Haller-Dintelmann, J. Rehberg: Maximal parabolic regularity for divergence operators on distribution spaces. In: J. Escher et al. (eds.): Parabolic Problems, The Herbert Amann Festschrift, vol. 80 of Progress in Nonlinear Differential Equations and Their Applications, pp. 313-341, Birkhäuser, Basel, 2011.

[32] D. Henry: Geometric theory of semilinear parabolic equations. Vol 840 of Lecture Notes in Mathematics, Springer-Verlag, Berlin-New York, 1981.

[33] R. Herzog, C. Meyer, G. Wachsmuth: Integrability of displacement and stresses in linear and nonlinear elasticity with mixed boundary conditions. J. Math. Anal. Appl. 382 (2011), no. 2, 802-813.

[34] D. Hömberg, K. Krumbiegel, J. Rehberg: Optimal control of a parabolic equation with dynamic boundary condition. Appl. Math. Optim. 67 (2013), no. 1, 3-31.

[35] D. Hömberg, C. Meyer, J. Rehberg, W. Ring: Optimal control for the thermistor problem. SIAM J. Control Optim. 48 (2009/10), no. 5, 3449-3481.

[36] D. Jerison, C. Kenig: The inhomogeneous Dirichlet problem in Lipschitz domains. J. Funct. Anal. 130 (1995), no. 1, 161-219.

[37] P.W. Jones: Quasiconformal mappings and extendability of functions in Sobolev spaces. Acta Math. 147 (1981), 71-88.

[38] A. Jonsson, H. Wallin: Function spaces on subsets of $\mathbb{R}^{n}$, Math. Rep. 2 (1984), no. 1.

[39] A. Jüngel: Regularity and uniqueness of solutions to a parabolic system in nonequilibrium thermodynamics. Nonlinear Anal. 41 (2000), no. 5-6, 669-688.

[40] H.-C. Kaiser, H. Neidhardt, J. Rehberg: Classical solutions of drift-diffusion equations for semiconductor devices: The 2D case. Nonlinear Anal. 71 (2009) 1584-1605.

[41] T. Kato: Perturbation theory for linear operators. Reprint of the corr. print. of the 2nd ed., Classics in Mathematics, Springer-Verlag, Berlin, 1980.

[42] D. Knees, P. Neff: Regularity up to the boundary for nonlinear elliptic systems arising in time-incremental infinitesimal elasto-plasticity. SIAM J. Math. Anal. 40 (2008), no. 1, 21-43.

[43] D. Knees, R. Rossi, C. Zanini: A vanishing viscosity approach to a rate-independent damage model, Math. Models Methods Appl. Sci. 23 (2013), no. 4, 565-616.

[44] H. König: Eigenvalue Distribution of Compact Operators. Vol. 16 of Operator Thoery: Advances and Applications, Birkhäuser, Basel, 1986.

[45] S.G. Krein, Yu.I. Petunin: Scales of Banach spaces. Russ. Math. Surv. 21 (1966), no. 2, 85-160.

[46] J.J. Liu, J. Cheng, G. Nakamura: Reconstruction and uniqueness of an inverse scattering problem with impedance boundary. Sci. China Ser. A 45 (2003), no. 11, 1408-1419.

[47] A. Lunardi: Analytic semigroups and optimal regularity in parabolic problems. Vol. 16 of Progress in Nonlinear Differential Equations and their Applications, Birkhäuser Verlag, Basel, 1995.

[48] G.J. Martin: Quasiconformal and bi-Lipschitz homeomorphisms, uniform domains and the quasihyperbolic metric. Trans. Amer. Math. Soc. 292 (1985), no. 1, 169-191.

[49] O. Martio: Definitions for uniform domains. Ann. Acad. Sci. Fenn., Ser. A I Math. 5 (1980), no. 1, 197-205.

[50] O. Martio, J. Sarvas: Injectivity theorems in plane and space. Ann. Acad. Sci. Fenn., Ser. A I Math. 4 (1979) 383-401.

[51] V. Maz'ya: Sobolev Spaces. Springer, Berlin, 1985.

[52] V. Maz'ya, J. Elschner, J. Rehberg, G. Schmidt: Solutions for quasilinear nonsmooth evolution systems in $L^{p}$. Arch. Ration. Mech. Anal. 171 (2004), no. 2, 219-262.

[53] N. Meyers: An $L^{p}$-estimate for the gradient of solutions of second order elliptic divergence equations. Ann. Scuola Norm. Sup. Pisa (3) 17 (1963) 189-206.

[54] A. Mielke: On the energetic stability of solitary water waves R. Soc. Lond. Philos. Trans. Ser. A Math. Phys. Eng. Sci. 360 (2002), no. 1799, 2337-2358.

[55] I. Mitrea, M. Mitrea: The Poisson problem with mixed boundary conditions in Sobolev and Besov spaces in non-smooth domains. Trans. Amer. Math. Soc. 359 (2007), no. 9, 4143-4182.

[56] P. Neff: The Cosserat couple modulus for continuous solids is zero viz the linearized Cauchy-stress tensor is symmetric. ZAMM, Z. Angew. Math. Mech. 86 (2006), no. 11, 892-912.

[57] S. Selberherr: Analysis and simulation of semiconductor devices. Springer, Wien, 1984.

[58] E. Shamir: Regularization of mixed second-order elliptic problems. Israel J. Math. 6 (1968) 150-168.

[59] I. Sneiberg: Spectral properties of linear operators in families of Banach spaces. Mat. Issled. 9 (1974) 214-229.

[60] I. E. Tamm: Fundamentals of the theory of electricity. Mir Publishers, Moscow, 1979.

[61] H. Triebel: Interpolation theory, function spaces, differential operators. North Holland Publishing Company, Amsterdam-New York-Oxford, 1978.

[62] J. Väisälä: Uniform domains. Tohoku Math. J. (2) 40 (1988), no. 1, 101-118.

[63] A. Vignati, M. Vignati: Spectral theory and complex interpolation. J. Funct. Anal. 80 (1988), no. 2, 383-397.

[64] S. Yang: A Sobolev extension domain that is not uniform. Manuscr. Math. 120 (2006), no. 2, 241-251.

[65] W. Ziemer: Weakly Differentiable Functions. Springer, 1989. 
Technische Universität Darmstadt, Fachbereich Mathematik, Schlossgartenstr. 7, D-64298 DarmSTADT, Germany

E-mail address: haller@mathematik.tu-darmstadt.de

Umea universitet SE-901 87 Umea Sverige

E-mail address: alf.jonsson@math.umu.se

Weierstrass Institute for Applied Analysis and Stochastics, Mohrenstr. 39, D-10117 Berlin, GerMANY

E-mail address: dorothee.knees@wias-berlin.de

Weierstrass Institute for Applied Analysis and Stochastics, Mohrenstr. 39, D-10117 Berlin, GerMANY

E-mail address: rehberg@wias-berlin.de 